\title{
Systematic review of the validity and reliability of consumer-wearable activity trackers
}

\author{
Kelly R. Evenson ${ }^{1,2^{*}}$, Michelle M. Goto ${ }^{1}$ and Robert D. Furberg ${ }^{2}$
}

\begin{abstract}
Background: Consumer-wearable activity trackers are electronic devices used for monitoring fitness- and other health-related metrics. The purpose of this systematic review was to summarize the evidence for validity and reliability of popular consumer-wearable activity trackers (Fitbit and Jawbone) and their ability to estimate steps, distance, physical activity, energy expenditure, and sleep.
\end{abstract}

Methods: Searches included only full-length English language studies published in PubMed, Embase, SPORTDiscus, and Google Scholar through July 31, 2015. Two people reviewed and abstracted each included study.

Results: In total, 22 studies were included in the review (20 on adults, 2 on youth). For laboratory-based studies using step counting or accelerometer steps, the correlation with tracker-assessed steps was high for both Fitbit and Jawbone (Pearson or intraclass correlation coefficients $(C C)>=0.80$ ). Only one study assessed distance for the Fitbit, finding an over-estimate at slower speeds and under-estimate at faster speeds. Two field-based studies compared accelerometry-assessed physical activity to the trackers, with one study finding higher correlation (Spearman CC 0.86, Fitbit) while another study found a wide range in correlation (intraclass CC 0.36-0.70, Fitbit and Jawbone). Using several different comparison measures (indirect and direct calorimetry, accelerometry, self-report), energy expenditure was more often under-estimated by either tracker. Total sleep time and sleep efficiency were overestimated and wake after sleep onset was under-estimated comparing metrics from polysomnography to either tracker using a normal mode setting. No studies of intradevice reliability were found. Interdevice reliability was reported on seven studies using the Fitbit, but none for the Jawbone. Walking- and running-based Fitbit trials indicated consistently high interdevice reliability for steps (Pearson and intraclass CC 0.76-1.00), distance (intraclass CC 0.90-0.99), and energy expenditure (Pearson and intraclass CC 0.71-0.97). When wearing two Fitbits while sleeping, consistency between the devices was high.

Conclusion: This systematic review indicated higher validity of steps, few studies on distance and physical activity, and lower validity for energy expenditure and sleep. The evidence reviewed indicated high interdevice reliability for steps, distance, energy expenditure, and sleep for certain Fitbit models. As new activity trackers and features are introduced to the market, documentation of the measurement properties can guide their use in research settings.

Keywords: Distance, Energy expenditure, Fitbit, Intervention, Jawbone, Measurement, Physical activity, Sleep, Steps, Walking

\footnotetext{
* Correspondence: kelly_evenson@unc.edu

${ }^{1}$ Department of Epidemiology, Gillings School of Global Public Health,

University of North Carolina-Chapel Hill, 137 East Franklin Street, Suite 306,

Chapel Hill 27514NC, USA

${ }^{2}$ RTI International, Research Triangle Park, NC, USA
}

(C) 2015 Evenson et al. Open Access This article is distributed under the terms of the Creative Commons Attribution 4.0 International License (http://creativecommons.org/licenses/by/4.0/), which permits unrestricted use, distribution, and reproduction in any medium, provided you give appropriate credit to the original author(s) and the source, provide a link to the Creative Commons license, and indicate if changes were made. The Creative Commons Public Domain Dedication waiver (http://creativecommons.org/publicdomain/zero/1.0/) applies to the data made available in this article, unless otherwise stated. 


\section{Background}

Consumer wearable devices are a popular and growing market for monitoring physical activity, sleep, and other behaviors. The devices helped to grow what is known as the Quantified Self movement, engaging those who wish to track their own personal data to optimize health behaviors [1]. A subset of consumer wearable devices used for monitoring physical activity- and fitness-related metrics are referred to as "activity trackers" or "fitness trackers" [2]. Their popularity has risen as they have become more affordable, unobtrusive, and useful in their application. An activity tracker can provide feedback and offer interactive behavior change tools via a mobile device, base station, or computer for long-term tracking and data storage $[3,4]$. The trackers enable self-monitoring towards daily or longer-term goals (such as a goal to walk a certain distance over time) and can be used to compare against one's peers or a broader community of users, both of which are advantageous mediators to increasing walking and overall physical activity $[3,5]$.

A national United States (US) survey completed in 2012 indicated $69 \%$ of adults tracked at least one health indicator for themselves, a family member, or friend using a tracking device (such as an activity tracker), paper tracking, or another method [6]. From this survey, $60 \%$ of adults reported tracking weight, diet, or exercise. Those who tracked weight, diet, or exercise were similar by gender, but more likely to be non-Hispanic White or African American, older, and have at least a college degree compared to Hispanics, younger ages, and those with less than a college degree, respectively. Among those who tracked at least one health behavior or condition, $21 \%$ used some form of technology to track the health data. Also among this group, $46 \%$ indicated that tracking changed their overall approach to maintaining their health or the health of the person they cared for, $40 \%$ indicated that it led them to ask a doctor new questions or obtain a second opinion, and $34 \%$ indicated that it affected a decision about how to treat an illness or condition.

Activity trackers are being used not only in the consumer market but also in research studies. Physical activity-related interventions are using activity trackers for self-monitoring, reinforcement, goal-setting, and measurement (examples among adults [4, 7-11] and youth [12]). Before more widespread use of these trackers occurs in research studies, for either intervention or measurement purposes, it is important to establish their validity and reliability.

The purpose of this review was to summarize the evidence for validity and reliability of the most popular consumer-wearable activity trackers. Among a variety of trackers on the market, approximately 3.3 million sold between April 2013 to March 2014, with $96 \%$ made by Fitbit (67\%), Jawbone (18\%), and Nike (11\%) [2]. Since
Nike discontinued the sale of Fuelbands in 2014, our focus for this review was on activity trackers made by Fitbit and Jawbone. Before conducting the review, we searched company websites for documentation on the accuracy of measuring steps, distance, physical activity, energy expenditure, and sleep. The Fitbit company indicated that after multiple internal studies, they had "tuned the accuracy of the Fitbit tracker step counting functionality over hundreds of tests with multiple body types. All Fitbit trackers should be 95-97\% accurate for step counting when worn as recommended" [13]. However, no other information was provided to document the accuracy of steps, nor the other measures we reviewed. The Jawbone company indicated that "while variations in user, terrain, and activity conditions can influence specific calculations, testing has shown UP to provide industry-leading accuracy in tracking activity and sleep" [14]. Similarly, no other details were provided of how accuracy was determined. Therefore, we focused our search on the ability of these trackers to estimate steps, distance, physical activity, energy expenditure, and sleep. For each study included in the review, we also abstracted information on the tracker's feasibility of use.

\section{Methods}

\section{Literature search}

Searches of PubMed, Embase, and SPORTDiscus were conducted to include only full-length studies published in English language journals through July 31, 2015. No start date was imposed in the search. If a publication was available online first before print, we attempted to obtain a copy; thus, some publications were officially published after July 31, 2015 but were available in the databases during our search period. Two separate searches were performed for the two activity trackers.

(1) (Fitbit) AND (validity OR validation OR validate OR comparison OR comparisons OR comparative OR reliability OR accuracy)

(2) (Jawbone) AND monitor AND (validity OR validation $\mathrm{OR}$ validate $\mathrm{OR}$ comparison $\mathrm{OR}$ comparisons OR comparative OR reliability OR accuracy)

The term "monitor" was added to the Jawbone search to reduce the number of dental-related articles retrieved. In addition, we reviewed Google Scholar similarly (same search terms, dates, only English language journals) and the reference lists of included studies for publications missed by the searches. We excluded abstracts (examples $[15,16])$ and conference proceedings (example [17]). We also excluded studies focused on special populations, such as stroke and traumatic brain injury [18], chronic obstructive pulmonary disease [19], amputation [20], mental 
illness [21], or older adults in assisted living [22]. One study presented data on apparently healthy older adults without mobility impairments and those of similar ages with reduced mobility; therefore, we reported only on those without mobility impairments [23].

\section{Abstraction and analysis}

First, we documented descriptive information on the activity trackers (models, release date, placement, size, weight, and cost) through internet searches conducted from May-July 2015. Second, an abstraction tool used for this review was expanded from a tool initially created by De Vries et al. [24] to document study characteristics and measurement properties of the activity trackers. Specifically, we extracted information on the study population, protocol, statistical analysis, and results related to validity, reliability, and feasibility. We also extracted any information provided by the studies on items entered into the activity tracker user account settings. A primary reviewer extracted details and a second reviewer checked each entry. Discrepancies in coding were resolved by consensus. For any abstracted information that was missing from the publication, we attempted to contact at least one author to obtain the information. Summary tables were created from the abstracted information.

Validity of the activity trackers included [25]:

- Criterion validity: comparing the trackers to a criterion measure of steps, distance traveled, physical activity, energy expenditure, and sleep.

- Construct validity: comparing the trackers to other constructs that should track or correlate positively (convergent validity) or negatively (divergent validity).

Reliability of the activity trackers included [25]:

- Intradevice reliability: test-retest results indicating consistency within the same tracker. This can be conducted in the lab (such as on a shaker table).

- Interdevice reliability: results indicating consistency across the same brand/type of tracker measured at the same time and worn in the same location. This can be assessed during activities performed in the laboratory or while free-living.

We interpreted the correlation coefficients (CC) using the following ratings: $0-<0.2$ poor, $0.2-<0.4$ fair, $0.4-<$ 0.6 moderate, $0.6-<0.8$ substantial, and $0.8-<1.0$ almost perfect [26]. Feasibility assessment included how much missing or lost data occurred and any feedback on wearing the trackers by participants.

\section{Results}

$>$ Through the systematic search, 67 records were identified, 39 were screened, and 22 were included in the review that reported on the validity or reliability of any Fitbit or Jawbone tracker. The Preferred Reporting Items for Systematic Reviews and Meta-Analyses (PRISMA) $[27,28]$ figure displays the detailed results from the search (Additional file 1). Twenty studies reported on at least one type of Fitbit tracker [15, 23, 29-46] and eight reported on at least one type of Jawbone tracker [30, 33, $35,40,42,45,47,48]$.

\section{Fitbit tracker}

The Fitbit company (San Francisco, CA; https://www.fitbit.com) has offered at least nine activity trackers since 2008 (Table 1). Depending on the type of activity tracker, the company recommends wearing them at the waist, wrist, pocket, or bra. The trackers contain a triaxial accelerometer and more recently an altimeter, heart rate, and global positioning system (GPS) monitor. Using proprietary algorithms, data from measures collected along with information input by the user can estimate steps, distance, physical activity, kilocalories, and sleep. Daylevel data is summarized and available to the consumer. Minute-level data (called "intraday") requires more effort to obtain, such as through the Fitbit API [32], and can be set at intervals of $1,5,10,15,20$, or $60 \mathrm{~min}$. Alternatively, data can be extracted using third-party service providers, such as Fitabase (Small Steps Labs LLC; https://www.fitabase.com), as was used in the study by Diaz et al. [15].

The Fitbit One updated the Fitbit Ultra in 2012, which in turn updated the Fitbit Classic in 2011, and all three are shaped similarly as a clip. The Fitbit Zip is teardropshaped and the Fitbit Flex is designed for the wrist. The following Fitbit trackers were explored for validity (Table 2):

(1)Classic worn at the waist $[29,31,39,41]$ and nondominant wrist [38];

(2)Ultra worn at the waist/hip [23, 29, 34, 36, 40], pants pocket [32, 36], dominant-handed wrist [23], non-dominant wrist [37], shirt collar [36], and bra [36];

(3) One worn at the waist [15, 30, 32, 33, 35, 42, 43, 46], pants pocket [43], and ankle [46];

(4)Zip worn at the waist [30, 33, 35, 44]; and

(5) Flex worn on the wrist $[15,30,45]$.

Reliability studies included the Classic worn at the waist [29] and non-dominant wrist [38]; the Ultra worn at the waist/hip [29, 36], pants pocket [32], and non-dominant wrist [37]; the One worn at the waist $[15,43]$ and pants pocket [43]; and the Flex worn on the wrist [15]. 
Table 1 Fitbit and Jawbone activity tracker characteristics (searched May-July 2015)

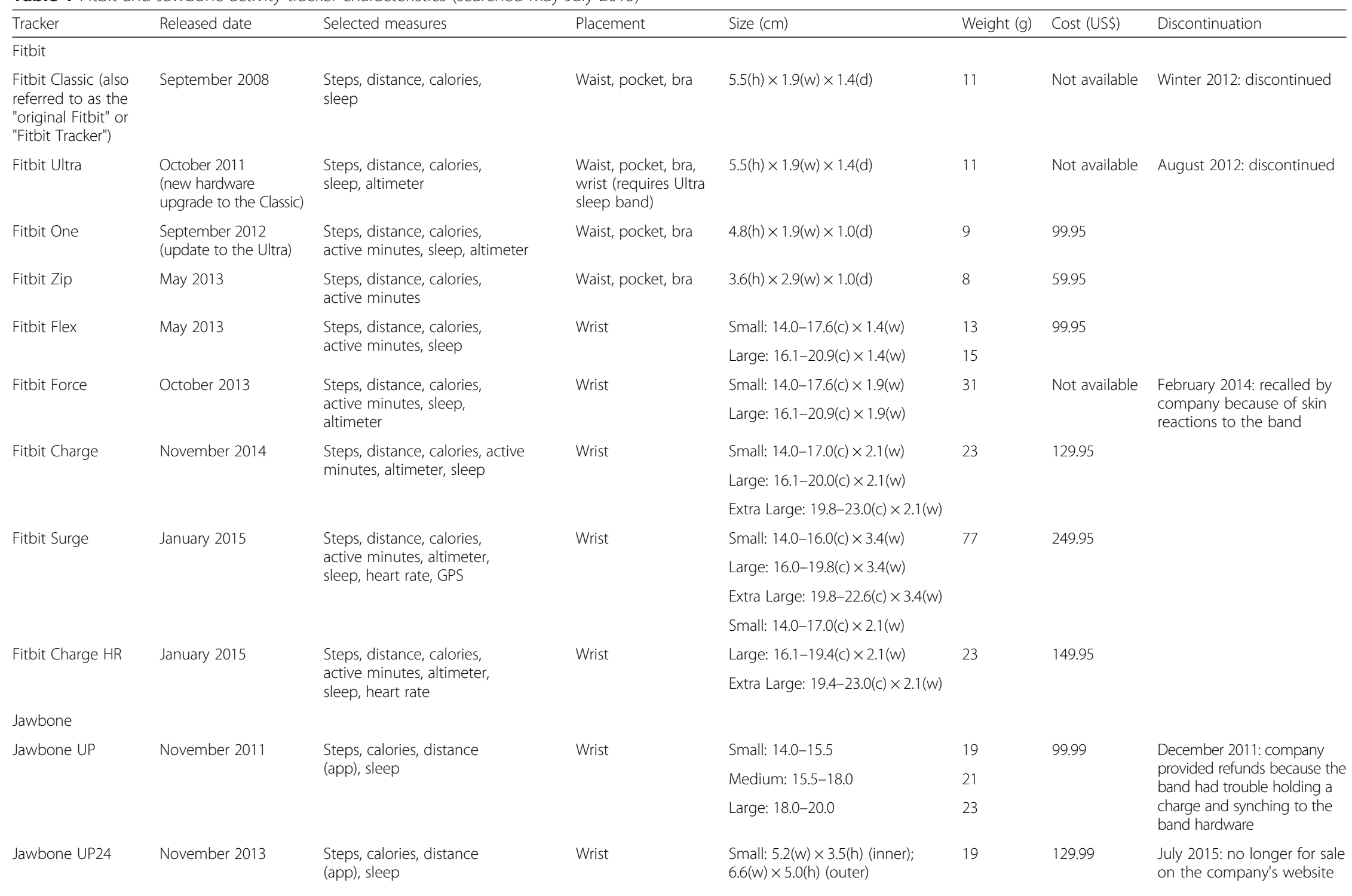


Table 1 Fitbit and Jawbone activity tracker characteristics (searched May-July 2015) (Continued)

\begin{tabular}{|c|c|c|c|c|c|c|}
\hline & & & & $\begin{array}{l}\text { Medium: } 6.3(\mathrm{w}) \times 4.0(\mathrm{~h}) \text { (inner); } \\
7.6(\mathrm{w}) \times 5.4(\mathrm{~h}) \text { (outer) }\end{array}$ & 22 & \\
\hline & & & & $\begin{array}{l}\text { Large: } 6.9(\mathrm{w}) \times 4.3(\mathrm{~h})(\text { inner) } \\
8.1(\mathrm{w}) \times 5.6(\mathrm{~h}) \text { (outer) }\end{array}$ & 23 & \\
\hline Jawbone UP MOVE & November 2014 & $\begin{array}{l}\text { Steps, calories, distance } \\
\text { (app), sleep }\end{array}$ & $\begin{array}{l}\text { Waist, pocket, bra, } \\
\text { wrist (requires } \\
\text { separate wrist strap) }\end{array}$ & 2.8 (diameter) × $1.0(\mathrm{~d})$ & 7 & 49.99 \\
\hline Jawbone UP2 & April 2015 & $\begin{array}{l}\text { Steps, calories, distance } \\
\text { (app), sleep }\end{array}$ & Wrist & $14.0-19.0(\mathrm{c}) \times 1.2(\mathrm{w})$ & 25 & 99.99 \\
\hline Jawbone UP3 & November 2014 & $\begin{array}{l}\text { Steps, calories, distance (app), } \\
\text { sleep, bioimpedance (heart } \\
\text { rate, respiration, galvanic skin } \\
\text { response), skin and ambient } \\
\text { temperature }\end{array}$ & Wrist & $14.0-19.0(\mathrm{c}) \times 1.2(\mathrm{w})$ & 29 & 179.99 \\
\hline Jawbone UP4 & July 2015 & $\begin{array}{l}\text { Steps, calories, distance (app), } \\
\text { sleep, bioimpedance (heart rate, } \\
\text { respiration, galvanic skin response), } \\
\text { skin and ambient temperature }\end{array}$ & Wrist & $14.0-19.0(\mathrm{c}) \times 1.2(\mathrm{w})$ & 29 & 199.99 \\
\hline
\end{tabular}

Abbreviations: c circumference, $d$ depth, GPS global positioning system, $h$ height, $w$ width 
Table 2 Fitbit and Jawbone studies of interdevice reliability and validity (listed by author's last name and publication year)

\begin{tabular}{|c|c|c|c|c|c|c|c|c|c|c|}
\hline \multirow[b]{2}{*}{ Motion sensor } & \multicolumn{2}{|l|}{ Interdevice reliability } & \multirow[b]{2}{*}{$\begin{array}{l}\text { Physical } \\
\text { activity }\end{array}$} & \multirow[b]{2}{*}{$\begin{array}{l}\text { Energy } \\
\text { expenditure }\end{array}$} & \multirow[b]{2}{*}{ Sleep } & \multicolumn{5}{|l|}{ Validity } \\
\hline & Steps & Distance & & & & Steps & Distance & Physical activity & Energy expenditure & Sleep \\
\hline \multicolumn{11}{|l|}{ Fitbit } \\
\hline $\begin{array}{l}\text { Fitbit Classic (also } \\
\text { referred to as the } \\
\text { "original Fitbit" or } \\
\text { "Fitbit Tracker") }\end{array}$ & Adam Noah 2013 [29] & & & $\begin{array}{l}\text { Adam Noah } \\
2013 \text { [29] }\end{array}$ & $\begin{array}{l}\text { Montgomery- } \\
\text { Downs } 2012 \\
\text { [38] }\end{array}$ & $\begin{array}{l}\text { Adam Noah } 2013 \\
\text { [29] }\end{array}$ & & & $\begin{array}{l}\text { Adam Noah } 2013 \text { [29]; } \\
\text { Dannecker } 2013 \text { [31]: } \\
\text { Sasaki } 2015 \text { [39]; } \\
\text { Stahl } 2014 \text { [41] }\end{array}$ & $\begin{array}{l}\text { Montgomery- } \\
\text { Downs } 2012 \text { [38] }\end{array}$ \\
\hline Fitbit Ultra & $\begin{array}{l}\text { Adam Noah } 2013 \text { [29]; } \\
\text { Dontje } 2015 \text { [32]; } \\
\text { Mammen } 2012 \text { [36] }\end{array}$ & & & $\begin{array}{l}\text { Adam Noah } \\
2013 \text { [29] }\end{array}$ & $\begin{array}{l}\text { Meltzer } 2015 \\
{[37]}\end{array}$ & $\begin{array}{l}\text { Adam Noah } 2013 \text { [29]; } \\
\text { Gusmer } 2014 \text { [34]; } \\
\text { Lauritzen } 2013 \text { [23]; } \\
\text { Mammen } 2012 \text { [36]; } \\
\text { Stackpool } 2014 \text { [40] }\end{array}$ & & & $\begin{array}{l}\text { Adam Noah } 2013 \text { [29]; } \\
\text { Gusmer } 2014 \text { [34]; } \\
\text { Stackpool } 2014 \text { [40] }\end{array}$ & Meltzer 2015 [37] \\
\hline Fitbit One & $\begin{array}{l}\text { Diaz } 2015 \text { [15]; } \\
\text { Takacs } 2014 \text { [43] }\end{array}$ & $\begin{array}{l}\text { Takacs } \\
2014\end{array}$ & & $\begin{array}{l}\text { Diaz } 2015 \\
{[15]}\end{array}$ & & $\begin{array}{l}\text { Case 2015 [30]; Diaz } \\
2015 \text { [15]; Ferguson } \\
2015 \text { [33]; Simpson } \\
2015 \text { [46]; Storm } \\
2015 \text { [42]; Takacs } \\
2014 \text { [43] }\end{array}$ & Takacs 2014 [43] & Ferguson 2015 [33] & $\begin{array}{l}\text { Diaz } 2015 \text { [15]; } \\
\text { Ferguson } 2015 \text { [33]; } \\
\text { Lee } 2014 \text { [35] }\end{array}$ & Ferguson 2015 [33] \\
\hline Fitbit Zip & & & & & & $\begin{array}{l}\text { Case } 2015 \text { [30]; } \\
\text { Ferguson } 2015 \text { [33]; } \\
\text { Tully } 2014 \text { [44] }\end{array}$ & & $\begin{array}{l}\text { Ferguson } 2015 \text { [33]; } \\
\text { Tully } 2014 \text { [44] }\end{array}$ & $\begin{array}{l}\text { Ferguson } 2015 \text { [33]; } \\
\text { Lee } 2014 \text { [35] }\end{array}$ & \\
\hline Fitbit Flex & Diaz 2015 [15] & & & $\begin{array}{l}\text { Diaz } 2015 \\
\text { [15] }\end{array}$ & & $\begin{array}{l}\text { Case } 2015 \text { [30]; } \\
\text { Diaz } 2015 \text { [15] }\end{array}$ & & & $\begin{array}{l}\text { Bai } 2015 \text { [45]; } \\
\text { Diaz } 2015 \text { [15] }\end{array}$ & \\
\hline \multicolumn{11}{|l|}{ Jawbone } \\
\hline Jawbone UP & & & & & & $\begin{array}{l}\text { Ferguson } 2015[33] ; \\
\text { Stackpool } 2014[40] ; \\
\text { Storm } 2014[42]\end{array}$ & & Ferguson 2015 [33] & $\begin{array}{l}\text { Ferguson } 2015 \text { [33]; } \\
\text { Lee } 2014 \text { [35]; } \\
\text { Stackpool } 2014 \text { [40] }\end{array}$ & $\begin{array}{l}\text { de Zambotti 2015a } \\
\text { [47]; de Zambotti } \\
\text { 2015b; Ferguson } \\
2015\end{array}$ \\
\hline Jawbone UP24 & & & & & & Case 2015 [30] & & & & Bai 2015 [45] \\
\hline
\end{tabular}

We found no studies for the Fitbit Force, Surge, Charge, or Charge HR, or the Jawbone UP MOVE, UP2, UP3, or UP4 


\section{Jawbone tracker}

The Jawbone company (San Francisco, CA; https://jawbone.com) has offered at least six activity trackers since 2011 (Table 1). Their trackers are worn at the wrist, with the exception of the UP MOVE tracker to be worn at the waist, pocket, or bra. The trackers contain a triaxial accelerometer, collecting data at $30 \mathrm{Hertz}$, and more recently bioelectrical impedance (for heart rate, respiration, and skin response), as well as both skin and ambient temperatures. Using proprietary algorithms, data from measures collected along with information input by the user can estimate steps, distance, physical activity, kilocalories, and sleep. Currently, only day-level data is available to the consumer.

The following two Jawbone trackers, both designed for the wrist, were explored for validity (Table 2):

(1)UP worn on the wrist [33, 35, 40, 42, 47, 48] and

(2)UP24 worn on the wrist [30, 45].

No Jawbone trackers were explored for reliability.

About half of the studies reported the data entered into the tracker user account [29, 33-35, 39, 41, 43], which was usually age, gender, height, and weight. One study also reported entering stride length [34], another study input handedness and smoking status [35], and another study used event markers to denote when an activity started and ended [39]. A sleep study indicated that they manually switched the band from active to sleep mode in conjunction with lights on/off [48]. Other studies did not report what data were input into the user account $[15,23,30-32,36-38,40,42,44-47]$.

\section{Description of studies}

Data collection was primarily conducted in the US, with one or two studies conducted in Australia [33], Canada [36, 43, 46], the Netherlands [32], Northern Ireland [44], Spain [23], and the United Kingdom [42] (Table 3). Studies usually included an apparently healthy sample and, where reported, almost all participants had a normal body mass index (BMI). Additionally, participants were $>=18$ years and mostly younger to middle age, except for one study focusing exclusively on adults $>=60$ years [41] and two studies on youth [37, 48]. Data were collected between 2010 [38] to 2015 [47].

\section{Validity}

All but one study (21/22) explored the validity of at least one type of activity tracker (Table 4). Sample sizes of the studies ranged from six [23] to 65 [48]. For any Fitbit tracker, validity was reported from 12 studies on steps $[15,23,29,30,33,34,36,40,42-44,46]$, one study on distance [43], two studies on physical activity [33, 44], ten studies on energy expenditure $[15,29,31,33-35$, $39-41,45]$, and three studies on sleep [33, 37, 38] (Table 2). For any Jawbone tracker, validity was reported from four studies on steps [30, 33, 40, 42], zero studies on distance, one study on physical activity [33], three studies on energy expenditure [33, 35, 40], and three studies on sleep [33, 47, 48]. The following sections detail the validity results for each of the five measures.

\section{Validity for steps}

The criterion measures for counting steps included comparisons against manual step counting, either in-person $[30,36,40]$ or with video recording $[15,23,43,46]$, or steps recorded by pedometers (Yamax CW-700 [44]) or accelerometers (Actical [29], ActiGraph GT1M [34], ActiGraph GT3X [44], ActiGraph GT3X+ [33], Body Media SenseWear [33], and Opal sensors [42]). Hipworn trackers generally outperformed wrist-worn trackers for step accuracy $[15,23,30,40]$. One study found less error for the ankle-worn One compared to the waist-worn One [46].

For laboratory-based studies using step counting as the criterion $[15,23,43]$, correlation with steps from the tracker was generally high (if reported, the mean correlations were $>=0.80$ ) for the Ultra (for most treadmill speeds [36]; for treadmill walking and elliptical but not for running or agility drills [40]), One [30, 43], Zip [30], and UP (for treadmill walking, running, and elliptical [40]) trackers. However, several studies indicated that the One [15], Flex [15, 30], Ultra (waist worn at slower walking speed $(2 \mathrm{~km} / \mathrm{h})$ and the pocket worn at faster speeds $(>=8 \mathrm{~km} / \mathrm{h})$ ) [36]), and UP24 [30] underestimated steps during treadmill walking and running.

For studies using accelerometry as the criterion, correlation with tracker steps was also generally high (if reported, the mean correlations were $>=0.80$ ) for the Classic [29], Ultra [29, 34], Zip [44], One [33], and UP [33] trackers. However, several studies indicated that the One [42], Flex [15, 30], UP [33](at slow walking speeds [42]), and UP24 [30] under-estimated steps during treadmill walking and running. In contrast, in a study of 21 participants wearing the One for 2 days without restrictions, compared to an accelerometer the tracker generally over-counted steps for the One (mean absolute difference 779 steps/day) [33]. In one free-living study, the researcher wore both the Ultra and a Yamax pedometer while seated in a car driving on paved roads for about $20 \mathrm{~min}$ [36]. During this time no steps were recorded for the Ultra, while the pedometer recorded three steps.

\section{Validity for distance}

Only one study explored the validity of distance walked using the treadmill distance as the criterion. Among 30 
Table 3 Characteristics of studies included in the systematic review (listed by author's last name and publication year)

\begin{tabular}{|c|c|c|c|c|c|c|}
\hline Author (year) & $\begin{array}{l}\text { Location of lab } \\
\text { or recruitment } \\
\text { area }\end{array}$ & $\begin{array}{l}\text { Sample size (for } \\
\text { validity and } \\
\text { reliability studies) }\end{array}$ & $\begin{array}{l}\text { Mean age } \\
(\mathrm{SD}) \text {, range }\end{array}$ & $\begin{array}{l}\text { Mean body mass index } \\
\text { (SD), range in kilograms/ } \\
\text { meters squared }\end{array}$ & $\begin{array}{l}\text { Data } \\
\text { collection } \\
\text { year(s) }\end{array}$ & Inclusion criteria \\
\hline $\begin{array}{l}\text { Adam Noah } \\
\text { (2013) [29] }\end{array}$ & $\begin{array}{l}\text { Northeastern } \\
\text { university, US }\end{array}$ & 16 and $23(V$ and $R)$ & $26.7(7.6)$ & Not reported & $2011-2012$ & $\begin{array}{l}\text { Apparently healthy participants, had to participate in moderate to vigorous physical } \\
\text { activity based on the International Physical Activity Questionnaire }(>=150 \text { minutes/ } \\
\text { week of moderate intensity or }>=75 \text { minutes/week of vigorous intensity) }\end{array}$ \\
\hline $\begin{array}{l}\text { Bai (2015) } \\
{[45]}\end{array}$ & Ames, lowa, US & $52(\mathrm{~V})$ & $18-65$ & $24.0,17.6-39.9$ & 2014 & Apparently healthy adults with no major surgeries in the past year \\
\hline $\begin{array}{l}\text { Case (2015) } \\
{[30]}\end{array}$ & $\begin{array}{l}\text { Philadelphia, } \\
\text { Pennsylvania, } \\
\text { US }\end{array}$ & $14(\mathrm{~V})$ & $28.1(6.2)$ & $22.7(1.5)$ & 2014 & Apparently healthy adults \\
\hline $\begin{array}{l}\text { Dannecker } \\
\text { (2013) [31] }\end{array}$ & $\begin{array}{l}\text { Fort Collins and } \\
\text { Denver, } \\
\text { Colorado, US }\end{array}$ & $19(\mathrm{~V})$ & $26.9(6.6)$ & $25.1(4.6)$ & 2010 & Apparently healthy adults, inactive to moderately active ( $<6$ hours/week of exercise) \\
\hline $\begin{array}{l}\text { de Zambotti } \\
\text { (2015a) [47] }\end{array}$ & $\begin{array}{l}\text { San Francisco, } \\
\text { California, US }\end{array}$ & $28(\mathrm{~V})$ & $50.1(3.9)$ & $24.6(3.6)$ & $\begin{array}{l}2014- \\
2015\end{array}$ & Perimenopausal women \\
\hline $\begin{array}{l}\text { de Zambotti } \\
\text { (2015b) [48] }\end{array}$ & $\begin{array}{l}\text { San Francisco, } \\
\text { California, US }\end{array}$ & $65(V)$ & $15.8(2.5)$ & $21.2(3.5)$ & 2014 & Apparently healthy without sleep disorders \\
\hline $\begin{array}{l}\operatorname{Diaz}(2015) \\
{[15]}\end{array}$ & $\begin{array}{l}\text { New York City, } \\
\text { New York, US }\end{array}$ & $23(\mathrm{~V}$ and $\mathrm{R})$ & $20-54$ & $19.6-29.9$ & $\begin{array}{l}2013- \\
2014\end{array}$ & Apparently healthy \\
\hline $\begin{array}{l}\text { Dontje } \\
\text { (2015)[32] }\end{array}$ & $\begin{array}{l}\text { Groningen, The } \\
\text { Netherlands }\end{array}$ & $1(\mathrm{R})$ & 46 & Not reported & 2012 & Not reported \\
\hline $\begin{array}{l}\text { Ferguson } \\
\text { (2015) [33] }\end{array}$ & $\begin{array}{l}\text { Adelaide, South } \\
\text { Australia }\end{array}$ & $21(\mathrm{~V})$ & $\begin{array}{l}32.8(10.2) \\
20-59\end{array}$ & $\begin{array}{l}27.3 \text { (3.2) male; } 25.5 \text { (5.2) } \\
\text { female }\end{array}$ & 2013 & Apparently healthy \\
\hline $\begin{array}{l}\text { Gusmer } \\
\text { (2014) [34] }\end{array}$ & $\begin{array}{l}\text { Minneapolis, } \\
\text { Minnesota, US }\end{array}$ & $32(\mathrm{~V})$ & $\begin{array}{l}21.1(1.7), 18- \\
29\end{array}$ & Not reported & 2012 & Apparently healthy \\
\hline $\begin{array}{l}\text { Lauritzen } \\
\text { (2013) [23] }\end{array}$ & Seville, Spain & $6(\mathrm{~V})$ & $\begin{array}{l}35.3(6.5), 24- \\
45\end{array}$ & Not reported & $\begin{array}{l}\text { not } \\
\text { reported }\end{array}$ & $\begin{array}{l}\text { Not reporting on sample with reduced mobility and no results on older sample with } \\
\text { normal mobility }\end{array}$ \\
\hline $\begin{array}{l}\text { Lee }(2014) \\
{[35]}\end{array}$ & Ames, lowa, US & $60(V)$ & $\begin{array}{l}24.2(4.7) \\
\text { female; } 28.6 \\
\text { (6.4) male }\end{array}$ & $\begin{array}{l}24.3(2.6), 19.528 .0 \text { male; } \\
21.8(2.7), 18.1-31.2 \text { female }\end{array}$ & 2013 & No major disease and nonsmokers \\
\hline $\begin{array}{l}\text { Mammen } \\
(2012)[36]\end{array}$ & Toronto, Canada & 10 (V)and 1 (R) & $\begin{array}{l}23.0(1.2), 20- \\
25\end{array}$ & $21.4(1.9)$ & $\begin{array}{l}2011- \\
2012\end{array}$ & Healthy young adults \\
\hline $\begin{array}{l}\text { Meltzer } \\
(2015)[37]\end{array}$ & $\begin{array}{l}\text { Birmingham, } \\
\text { Alabama, US }\end{array}$ & $63(\mathrm{~V})$ and $9(\mathrm{R})$ & $9.7(4.6), 3-17$ & Not reported & $\begin{array}{l}2012- \\
2013\end{array}$ & $\begin{array}{l}\text { Sample referred to clinic for sleep disordered breathing; results of polysomnography } \\
\text { indicated: } 61 \% \text { none, } 23 \% \text { mild, } 16 \% \text { moderate to severe }\end{array}$ \\
\hline $\begin{array}{l}\text { Montgomery- } \\
\text { Downs (2012) } \\
\text { [38] }\end{array}$ & $\begin{array}{l}\text { Morgantown, } \\
\text { West Virginia, } \\
\text { US }\end{array}$ & $24(\mathrm{~V})$ and $3(\mathrm{R})$ & $26.1,19-41$ & Not reported & 2010 & Healthy adults, no sleep disorders \\
\hline $\begin{array}{l}\text { Sasaki (2015) } \\
{[39]}\end{array}$ & $\begin{array}{l}\text { Amherst, } \\
\text { Massachusetts, } \\
\text { US }\end{array}$ & $20(V)$ & $24.1(4.5)$ & $23.9(2.9)$ & $\begin{array}{l}2011- \\
2012\end{array}$ & Apparently healthy \\
\hline $\begin{array}{l}\text { Simpson } \\
\text { (2015) [46] }\end{array}$ & $\begin{array}{l}\text { Vancouver, } \\
\text { Canada }\end{array}$ & $42(\mathrm{~V})$ & $73(6.9)$ & $26.1(4.6)$ & 2014 & $>=65$ years, able to walk unassisted \\
\hline
\end{tabular}


Table 3 Characteristics of studies included in the systematic review (listed by author's last name and publication year) (Continued)

\begin{tabular}{|c|c|c|c|c|c|c|}
\hline $\begin{array}{l}\text { Stackpool } \\
(2014)[40]\end{array}$ & $\begin{array}{l}\text { LaCrosse, } \\
\text { Wisconsin, US }\end{array}$ & $20(V)$ & $18-44$ & Not reported & 2013 & Healthy volunteers; all were recreationally active ( $2-5$ hours/week) \\
\hline $\begin{array}{l}\text { Stahl (2014) } \\
{[41]}\end{array}$ & $\begin{array}{l}\text { Morgantown, } \\
\text { West Virginia, } \\
\text { US }\end{array}$ & $10(\mathrm{~V})$ & $\begin{array}{l}63.8(3.2), 60- \\
68\end{array}$ & $24.5(4.2)$ & 2011 & $\begin{array}{l}\text { None noted; on average participants reported } 3 \text { chronic health conditions, no } \\
\text { functional limitations, and rated their health as "good" }\end{array}$ \\
\hline $\begin{array}{l}\text { Storm (2015) } \\
{[42]}\end{array}$ & $\begin{array}{l}\text { Sheffield, United } \\
\text { Kingdom }\end{array}$ & $16(V)$ & $28.9(2.7)$ & $23.5(2.3)$ & 2013 & $\begin{array}{l}\text { No reported impairment or morbidity that could interfere with physical activity } \\
\text { assessment }\end{array}$ \\
\hline $\begin{array}{l}\text { Takacs (2014) } \\
{[43]}\end{array}$ & $\begin{array}{l}\text { Vancouver, } \\
\text { Canada }\end{array}$ & $30(\mathrm{~V}$ and $\mathrm{R})$ & $29.6(5.7)$ & $22.7(3.0)$ & 2013 & $\begin{array}{l}\text { Able to walk on a treadmill for } 30 \text { min; no neurological, cognitive or musculoskeletal } \\
\text { disorders }\end{array}$ \\
\hline $\begin{array}{l}\text { Tully (2014) } \\
\text { [44] }\end{array}$ & $\begin{array}{l}\text { Belfast, Northern } \\
\text { Ireland }\end{array}$ & $42(V)$ & 43 & Not reported & 2013 & Apparently healthy staff of Queen's University Belfast \\
\hline
\end{tabular}

Abbreviations: $R$ reliability sample size, $S D$ standard deviation, US United States, $V$ validity sample size 
Table 4 Fitbit and Jawbone validity studies (listed by author's last name and publication year)

\begin{tabular}{|c|c|c|c|c|c|c|c|c|c|}
\hline \multirow[b]{2}{*}{ Author (year) } & \multicolumn{2}{|c|}{ Sample characteristics } & \multicolumn{3}{|l|}{ Tracker wearing protocol } & \multicolumn{3}{|c|}{ Measurements } & \multirow[t]{2}{*}{ Validity results } \\
\hline & $n$ & $\%$ female & Activity & $\begin{array}{l}\text { Lab/ } \\
\text { field }\end{array}$ & $\begin{array}{l}\text { Validity criterion (measure } \\
\text { assessed) }\end{array}$ & Type & Placement & Measures & \\
\hline \multirow[t]{2}{*}{$\begin{array}{l}\text { Adam Noah } \\
\text { et. al (2013) [29] }\end{array}$} & 16 & 38 & $\begin{array}{l}6 \text { min each of treadmill walking } \\
(3.5 \mathrm{mph}) \text {, walking with incline } \\
(3.5 \mathrm{mph} \text { at } 5 \% \text { ), jogging ( } 5.5 \\
\text { mph), and stair stepping ( } 30.5 \\
\text { centimeter step at } 96 \text { beats/min) }\end{array}$ & Lab & $\begin{array}{l}\text { Two Actical accelerometers } \\
\text { (steps), indirect calorimetry using } \\
\text { K4b2 Cosmed (EE) }\end{array}$ & $\begin{array}{l}\text { Ultra } \\
\text { (Fitbit) }\end{array}$ & $\begin{array}{l}\text { Waist (one } \\
\text { on each } \\
\text { side) }\end{array}$ & $\begin{array}{l}\text { Steps/min, } \\
\text { kilocalories/ } \\
\text { min }\end{array}$ & $\begin{array}{l}\text { Fitbit Ultra vs. Actical ICC: } \\
\text { average } 0.94, \text { range } 0.80-0.99 \\
\text { (steps); Fitbit Ultra vs. Cosmed } \\
\text { ICC: average 0.77, range } 0.58-0.87 \\
\text { (kilocalories) }\end{array}$ \\
\hline & 23 & 43 & & & & $\begin{array}{l}\text { Classic } \\
\text { (Fitbit) }\end{array}$ & $\begin{array}{l}\text { Waist (one } \\
\text { on each } \\
\text { side) }\end{array}$ & $\begin{array}{l}\text { Steps/min, } \\
\text { kilocalories/ } \\
\text { min }\end{array}$ & $\begin{array}{l}\text { Fitbit vs. Actical ICC: average } 0.93 \text {, } \\
\text { range } 0.82-0.98 \text { (steps); Fitbit vs. } \\
\text { Cosmed ICC: average } 0.74, \text { range } \\
0.18-0.72 \text { (kilocalories) }\end{array}$ \\
\hline \multirow[t]{2}{*}{$\begin{array}{l}\text { Bai et. al } \\
\text { (2015) [45] }\end{array}$} & 52 & 46 & $\begin{array}{l}20 \text { min sedentary, } 25 \text { min } \\
\text { treadmill at self-selected speed, } \\
25 \text { min resistance exercise }\end{array}$ & Lab & $\begin{array}{l}\text { Indirect calorimetry using } \\
\text { Oxycon Mobile (EE) }\end{array}$ & $\begin{array}{l}\text { Flex } \\
\text { (Fitbit) }\end{array}$ & Left wrist & $\begin{array}{l}\text { Kilocalories/ } \\
\text { 80- min trial }\end{array}$ & $\begin{array}{l}\text { Overestimated overall EE by } 20.4 \\
\text { kilocalories; Pearson CC } 0.78 \text {; overall } \\
\text { mean absolute error } 16.8 \%\end{array}$ \\
\hline & & & & & & $\begin{array}{l}\text { UP24 } \\
\text { (Jawbone) }\end{array}$ & Right wrist & & $\begin{array}{l}\text { Underestimated overall EE by } 23.1 \\
\text { kilocalories; Pearson CC } 0.77 \text {; overall } \\
\text { mean absolute error } 18.2 \%\end{array}$ \\
\hline \multirow[t]{4}{*}{$\begin{array}{l}\text { Case et. al } \\
\text { (2015) [30] }\end{array}$} & 14 & 71 & $\begin{array}{l}\text { Treadmill walking at } 3.0 \mathrm{mph} \text { for } \\
500 \text { and } 1500 \text { steps, each done } \\
\text { twice }\end{array}$ & Lab & Tally counter (steps) & $\begin{array}{l}\text { One } \\
\text { (Fitbit) }\end{array}$ & Waist & Steps/trial & $\begin{array}{l}500 \text { step trial ( } n=27 \text { observations) } \\
\text { mean } 498.6 \text { (SD 3.7); } 1500 \text { step trial } \\
(n=26 \text { observations) mean } 1497.0 \\
\text { (SD 10.7) }\end{array}$ \\
\hline & & & & & & $\begin{array}{l}\text { Zip } \\
\text { (Fitbit) }\end{array}$ & Waist & Steps/trial & $\begin{array}{l}500 \text { step trial ( } n=27 \text { observations) } \\
\text { mean } 498.6 \text { (SD 10.8); } 1500 \text { step } \\
\text { trial ( } n=27 \text { observations) mean } \\
1498.4 \text { (SD 10.4) }\end{array}$ \\
\hline & & & & & & $\begin{array}{l}\text { Flex } \\
\text { (Fitbit) }\end{array}$ & Wrist & Steps/trial & $\begin{array}{l}500 \text { step trial ( } n=28 \text { observations) } \\
\text { mean } 465.4 \text { (SD 92.1); } 1500 \text { step } \\
\text { trial ( } n=28 \text { observations) mean } \\
1378.0 \text { (SD 142.7) }\end{array}$ \\
\hline & & & & & & $\begin{array}{l}\text { UP24 } \\
\text { (Jawbone) }\end{array}$ & Wrist & Steps/trial & $\begin{array}{l}500 \text { step trial ( } n=28 \text { observations) } \\
\text { mean } 477.5 \text { (SD 102.1); } 1500 \text { step } \\
\text { trial ( } n=28 \text { observations) mean } \\
1477.0 \text { (SD 174.4) }\end{array}$ \\
\hline $\begin{array}{l}\text { Dannecker et. al } \\
\text { (2013) [31] }\end{array}$ & $\begin{array}{l}19 \text { (16 with } \\
\text { Fitbit data) }\end{array}$ & 47 (from $n=19$ ) & $\begin{array}{l}\text { Resting, supine, sitting, standing, } \\
\text { free living activity, and } 6 \text { random } \\
\text { activities out of } 8 \text { (walking ( } 2.5 \\
\text { mph, } 3.5 \mathrm{mph} \text {, or } 2.5 \mathrm{mph} \text { with } \\
2.5 \% \text { grade), stepping, } \\
\text { sweeping, cycling ( } 75 \text { watts), } \\
\text { standing, sitting }\end{array}$ & Lab & $\begin{array}{l}4 \mathrm{~h} \text { stay in whole room } \\
\text { calorimeter (EE) }\end{array}$ & $\begin{array}{l}\text { Classic } \\
\text { (Fitbit) }\end{array}$ & $\begin{array}{l}\text { Belt at } \\
\text { anterior } \\
\text { superior } \\
\text { iliac spine }\end{array}$ & $\begin{array}{l}\text { Total EE } \\
\text { during the } 3.5- \\
\text { h period while } \\
\text { in the room } \\
\text { calorimeter } \\
\text { (omitted first } \\
30 \text { minutes) }\end{array}$ & $\begin{array}{l}\text { Root-mean-square error of } \\
\text { tracker } 28.7 \% \text { or } 143 \text { kilocalories; } \\
\text { root-mean-square error of tracker } \\
\text { after labeling activities } 12.9 \% \text { or } \\
64 \text { kilocalories }\end{array}$ \\
\hline $\begin{array}{l}\text { de Zambotti } \\
\text { et. al (2015a) [47] }\end{array}$ & 28 & 100 & $\begin{array}{l}\text { One nights sleep }(n=10), 2 \\
\text { nights sleep }(n=18)\end{array}$ & Lab & Polysomnography (sleep) & $\begin{array}{l}\text { UP } \\
\text { (Jawbone) }\end{array}$ & $\begin{array}{l}\text { Non } \\
\text { dominant } \\
\text { wrist }\end{array}$ & $\begin{array}{l}\text { TST, sleep } \\
\text { onset latency, } \\
\text { WASO }\end{array}$ & $\begin{array}{l}\text { Overestimated TST by } 26.6 \pm \\
35.3 \mathrm{~min}(p<0.001) \text { and sleep } \\
\text { onset latency by } 5.2 \pm 9.6 \mathrm{~min}(p \\
=0.005) ; \text { underestimated WASO } \\
\text { by } 31.2 \pm 32.3 \mathrm{~min}(p<0.001)\end{array}$ \\
\hline
\end{tabular}


Table 4 Fitbit and Jawbone validity studies (listed by author's last name and publication year) (Continued)

de Zambotti et. 65

al (2015b) [48]

Diaz et. al

(2015) [15]

(1)

$48 \mathrm{~h}$ (including sleep, excluding showering) of free-living conditions, no activity restrictions/ guidelines

6 min each of treadmill walking Lab (1.9 mph, $3.0 \mathrm{mph}, 4.0 \mathrm{mph}$ ) and jogging (5.2 mph)

Lab Polysomnography (sleep)

UP

TST, sleep

efficiency,

sleep onset

latency,

WASO

Counting from a video recording One (steps), indirect calorimetry using (Fitbit) Ultima CPX (EE)

2 on right Steps/min,

hip, 1 on kilocalories/

eft hip min

Flex

(Fitbit)

1 on each

wrist

Steps/min kilocalories/ min

One
(Fitbit)

Right hip

(Fitbit)

Right hip MVPA min/ day,

kilocalories/

day, sleep

min/day

Steps/day, MVPA min/ day,

kilocalories/

day

(2015) [33]

BodyMedia SenseWear model

MF (steps, physical activity, EE

sleep): ActiGraph GT3X+ (steps,

physical activity)

UP

(Jawbone)

Left wrist

Steps/day,

MVPA min/

day,

kilocalories/

day, sleep

$\mathrm{min} /$ day

Ultra

0-min phases of treadmi

walking at slow and brisk

speeds $( \pm 10 \%$ of selfselected

Lab ActiGraph G1TM (steps), CPX

Ultima metabolic cart (EE)
Right hip

Steps/min,

kilocalories/

trial
Overestimated TST by $10.0 \mathrm{~min}$ $p<0.001)$, sleep efficiency by

$1.9 \%(p<0.001)$, and sleep onset atency by $1.3 \mathrm{~min}(p=0.33$ ); underestimated WASO by $10.6 \min (p<.001)$

Pearson CC 0.97-0.99 and mean difference -3.1 to -0.3 (steps); Pearson CC 0.86-0.87 (kilocalories) and mean difference -0.8 to 0.4 kilocalories

Pearson CC 0.77-0.85 and mean difference -26.3 to -2.9 (steps); Pearson CC 0.88 and mean difference -0.2 to 2.6 (kilocalories)

Pearson CC 0.99 (steps), 0.91 (MVPA), 0.76 (kilocalories), 0.92 (sleep); ICC 0.95 (steps), 0.46 (MVPA), 0.55 (kilocalories), 0.90 (sleep); mean absolute difference 779 (steps), 58.6 (MVPA), 349 (kilocalories), 23.0 (sleep); range of differences $=-890$ to 1849 (steps) 1.0 to 137.2 (MVPA), -1724 to -83 (kilocalories), 45 to 76 (sleep)

Pearson CC 0.99 (steps), 0.88 MVPA), 0.81 (kilocalories); ICC 0.98 (steps), 0.36 (MVPA), 0.57 (kilocalories); mean absolute difference 447 (steps), 89.8 (MVPA), 484 (kilocalories); range of differences -970 to 1596 (steps), 10.0 to 157.2 (MVPA) -1145 to 218 (kilocalories)

Pearson CC 0.97 (steps), 0.81 (MVPA), 0.74 (kilocalories), 0.89 (sleep); ICC 0.97 (steps), 0.70 (MVPA), 0.27 (kilocalories), 0.85 (sleep); mean absolute difference 806 (steps), 18.0 (MVPA), 866 (kilocalories), 22.0 (sleep); range of differences -1978 to 2252 (steps), -4.7 to 96.5 (MVPA), -1937 to -94 (kilocalories), - 31 to 132 (sleep)

Pearson CC: slow walk: 0.97 (steps: mean 105.3 ActiGraph vs. 105.9 Ultra), 0.69 (kilocalories: mean 100.9 cart vs. 88.0 Ultra); 
Table 4 Fitbit and Jawbone validity studies (listed by author's last name and publication year) (Continued)

Lauritzen et. al 6
(2013) [23]

[35]

Mammen et. al 10

(2012) [36] 20-meter walk at participant's normal pace

3 activities that were all 5 min in length except for treadmill ( 3 min each) totalling 69 minutes
Counting from a video recording Ultra (steps)

Oxycon Mobile (EE); ActiGraph GT3X+ worn on hip, applied Sasaki et al. 2011 [39] algorithm (EE)

One min on the treadmill at
each of 8 speeds ( 4 walking and 4 running)

pocket on leg, 1 on wrist of dominant hand

brisk walking: 0.996 (steps: mean 142 ActiGraph vs. 113.9 Ultra) 0.94 (kilocalories: mean 121.9 cart vs.100.9 Ultra)

1 on belt/ Steps/20-min Hip error $2.9 \%$ (SD $2.3 \%$ ); wrist pants trial error $31.3 \%$ (SD $30.7 \%$ )

(Jawbone)

Left wris

(a)

Kilocalories/

trial

(Fitbit)

Zip (Fitbit)

Waist

Kilocalories/

trial

Kilocalories/

trial

Mean absolute error $10.4 \%$; Pearson CC 0.81; root-meansquare error 40.1; did not fall in $90 \%$ equivalence interval; systematic bias with slope -0.22 comparing One $(x)$ to Oxycon $(y)$; Pearson CC to ActiGraph 0.80

Mean absolute error $10.1 \%$; Pearson CC 0.81; root-mean-square error 40.8; fell within $90 \%$ equivalence interval from measured $\mathrm{EE}$ systematic bias with slope - 0.29 comparing Zip (x) to Oxycon (y); Pearson CC to ActiGraph 0.77

Mean absolute error $12.2 \%$; Pearson CC 0.74; root-meansquare error 45.8; did not fall in $90 \%$ equivalence interval; no systematic direction of bias with slope - 0.03 comparing UP $(x)$ to Oxycon (y); Pearson CC to ActiGraph 0.65

Ultra Waist, Steps/tria (Fitbit) inside the pants pocket, shirt collar (men) or bra

(women)

Waist-worn Ultra under counted at $2 \mathrm{~km} /$ hour (31 steps/min; $p<$ $0.05)$ but had similar counts at $>$ $=3 \mathrm{~km} /$ hour. Pocket- worn Ultra under counted during running $(10,19,34,38$ steps/min at $8,9,10$ and $11 \mathrm{~km} /$ hour, respectively; $p<$ $0.05)$, but recorded similar counts when walking $(2,3,4.5$, and $6 \mathrm{~km} /$ hour). Similar counts across walk/ run trials for collar-(males) or bra(females) worn Ultras. 
Table 4 Fitbit and Jawbone validity studies (listed by author's last name and publication year) (Continued)

(2015) [37]
51

40

Downs et. al

(2012) [38]

24
One night's sleep

P

Polysomnography (sleep)

One night's sleep

Sasaki et. al
(2015) [39]

50

Simpson et. al

(2015) [46]

74

8 trials of walking 15 meters (self selected speed and 0.3 $0.9 \mathrm{~m} / \mathrm{s}$ at 0.1 increments)

Visit 1: 6 min each of treadmill
walking ( 3.0 at $5 \%$ and 4.0 at $5 \%$ ) and jogging (5.5 mph)

three trials: visit 2. 6 min each

of household activities (choice

from 3 activity routines)
Lab Polysomnography (sleep)

(Fitbit)

dominant

efficiency

Lab Oxycon Mobile (EE)

Classic
(Fitbit)

Belt around waist in

line with

axillary line

Lab

Counting from a video recording One (steps)

(Fitbit)

Right waist
right ankle

elliptical cross-training, agility-

related exercises
Lab Manually counting (steps);

Oxycon Mobile (EE)
Ultra

(Fitbit)
20 min each of: treadmil

walking, treadmill running,

Hip

Stackpool et.
(2014) [40]
Normal mode overestimated TST by 41 minutes and sleep efficiency by $8 \%$, underestimated WASO by 32 minutes; $87 \%$ sensitivity, $52 \%$ specificity, $84 \%$ accuracy. Sensitive mode underestimated TST by 105 minutes and sleep efficiency by $21 \%$ and overestimated WASO by 106 minutes; $70 \%$ sensitivity, $79 \%$ specificity, 71 \% accuracy.

Polysomnography recorded 465.0 min (SD 48.4) with $79.5 \%$ sleep efficiency and $370.9 \mathrm{~min}$ (SD 70.3) TST; Fitbit measured $94.0 \%$ sleep efficiency and 438.0 min TST; Fitbit

overestimated sleep efficiency compared to polysomnography by $14.5 \%$ (SD $10.7 \%$ ) and overestimated TST by mean $67.1 \mathrm{~min}$ (SD 51.3).

Total EE (rest Pearson CC 0.86; systematic underestimation of EE by the $1.0 \mathrm{kcals} / 6 \mathrm{~min}$ for 6 of 15 activities the Fitbit significantly underestimated EE (stairs, cycling, laundry, raking, treadmill $3.0 \mathrm{mph}$ with $5 \%$ grade, treadmill 4.0 mph with $5 \%$ grade) and 1 of 15
activities the Fitbit significantly overestimated EE (carrying. groceries)

$\%$ error: $0.3 \mathrm{~m} / \mathrm{s}$ : ankle 14.5 , waist 98.4; $0.4 \mathrm{~m} / \mathrm{s}$ : ankle 5.9 , waist 82.0 $0.5 \mathrm{~m} / \mathrm{s}$ : ankle 4.1 , waist 40.4 ; $0.6 \mathrm{~m} / \mathrm{s}$ : ankle 3.2, waist 21.6; $0.7 \mathrm{~m} / \mathrm{s}$ : ankle 2.5 , waist $10.5 ; 0.8 \mathrm{~m} / \mathrm{s}$ : ankle 2.8 , waist 7.0 ; $0.9 \mathrm{~m} / \mathrm{s}$ : ankle 2.8, waist 5.6; Bland Altman mean difference -0.4 to 5.7 steps for ankle and 1.4 to 48.0 for waist

Pearson CC: treadmill walking (0.99 steps, 0.24 kilocalories), treadmill running (0.44 steps, 0.63 kilocalories), elliptical (0.99 steps, 0.47 kilocalories), agility (0.47 steps, 0.67 kilocalories) 
Table 4 Fitbit and Jawbone validity studies (listed by author's last name and publication year) (Continued)

\begin{tabular}{|c|c|c|c|c|c|c|c|c|c|}
\hline \multirow[b]{2}{*}{$\begin{array}{l}\text { Stahl and } \\
\text { Insana (2014) } \\
\text { [41] }\end{array}$} & \multirow[b]{2}{*}{10} & \multirow[b]{2}{*}{30} & \multirow[b]{2}{*}{$\begin{array}{l}\text { During waking hours for } 10 \\
\text { consecutive days }\end{array}$} & \multirow[b]{2}{*}{ Field } & \multirow[b]{2}{*}{$\begin{array}{l}\text { Self-reported estimation of } \\
\text { expended kilocalories/week from } \\
\text { CHAMPS questionnaire (EE). } \\
\text { Note: kilocalories/week divided } \\
\text { by } 7 \text { to obtain kilocalories/day; } \\
\text { then basal metabolic rate was } \\
\text { added to the kilocalories/day. }\end{array}$} & \multirow[b]{2}{*}{$\begin{array}{l}\text { Classic } \\
\text { (Fitbit) }\end{array}$} & \multirow[b]{2}{*}{ Waist } & \multirow[b]{2}{*}{$\begin{array}{l}\text { Kilocalories/ } \\
\text { day }\end{array}$} & \multirow[b]{2}{*}{$\begin{array}{l}\text { Pearson CC } 0.61 \text {; Fitbit } \\
\text { underestimated by a mean of } \\
195.0 \text { kilocalories/day; } 70 \% \text { of } \\
\text { participant's data were within } 1 \\
\text { SD and } 100 \% \text { were within } 2 \text { SD }\end{array}$} \\
\hline & & & & & & & & & \\
\hline \multirow[t]{2}{*}{$\begin{array}{l}\text { Storm et. al } \\
\text { (2015) [42] }\end{array}$} & \multirow[t]{2}{*}{16} & \multirow[t]{2}{*}{38} & \multirow[t]{2}{*}{$\begin{array}{l}\text { 11-min walking protocol } \\
\text { (included indoor and outdoor } \\
\text { walking and steps) repeated at } \\
\text { self-selected natural, slow, and } \\
\text { fast speeds }\end{array}$} & \multirow[t]{2}{*}{ Lab } & \multirow[t]{2}{*}{$\begin{array}{l}\text { OPAL sensors placed on each } \\
\text { ankle (steps) }\end{array}$} & $\begin{array}{l}\text { One } \\
\text { (Fitbit) }\end{array}$ & Left waist & $\begin{array}{l}\text { Steps/11-min } \\
\text { trial }\end{array}$ & $\begin{array}{l}1.1 \% \text { self-selected walk, } 1.0 \% \\
\text { fast walk; limits of agreement } 15 \\
\pm 35 \text { steps; under estimated for } \\
\text { slow walk ( }-25 \text { mean steps), self- } \\
\text { selected walk ( }-12 \text { mean steps), } \\
\text { fast walk ( }-9 \text { mean steps) }\end{array}$ \\
\hline & & & & & & $\begin{array}{l}\text { UP } \\
\text { (Jawbone) }\end{array}$ & Right wrist & $\begin{array}{l}\text { Steps/11-min } \\
\text { trial }\end{array}$ & $\begin{array}{l}\text { Mean absolute error } 10.1 \% \text { slow } \\
\text { walk, } 2.5 \% \text { self-selected walk, } \\
2.1 \% \text { fast walk; limits of agree- } \\
\text { ment } 16 \pm 135 \text {; under estimated } \\
\text { for slow walk ( }-35 \text { mean steps), } \\
\text { self-selected walk ( }-4 \text { mean } \\
\text { steps), fast walk ( }-9 \text { mean steps) }\end{array}$ \\
\hline $\begin{array}{l}\text { Takacs et. al } \\
\text { (2014) [43] }\end{array}$ & 30 & 50 & $\begin{array}{l}5 \text { min each of treadmill walking } \\
(0.90,1.12,1.33,1.54,1.78 \\
\text { meters/second })\end{array}$ & Lab & $\begin{array}{l}\text { Motion capture system and } \\
\text { manually counting (steps); } \\
\text { treadmill output (distance) }\end{array}$ & $\begin{array}{l}\text { One } \\
\text { (Fitbit) }\end{array}$ & $\begin{array}{l}1 \text { right hip, } \\
1 \text { left hip, } 1 \\
\text { in front } \\
\text { pocket of } \\
\text { the } \\
\text { dominant } \\
\text { leg }\end{array}$ & $\begin{array}{l}\text { Steps/trial, } \\
\text { distance/trial }\end{array}$ & $\begin{array}{l}\text { Steps: no significant difference } \\
(p>0.05) \text { between observed and } \\
\text { One step counts at any of the } 3 \\
\text { locations, ICC } 0.97-1.00 \text {, relative } \\
\text { error }<1.3 \% \text {. Distance: significant } \\
\text { differences between observed } \\
\text { and One distance, ICC } 0.0-0.05 \text {, } \\
\text { relative error } 5.0-39.6 \% \text {. }\end{array}$ \\
\hline $\begin{array}{l}\text { Tully et. al } \\
\text { (2014) [44] }\end{array}$ & 42 & 60 & $\begin{array}{l}7 \text { days of free-living wear ex- } \\
\text { cluding water activities and } \\
\text { sleep }\end{array}$ & Field & $\begin{array}{l}\text { ActiGraph GT3X and Yamax } \\
\text { CW700 pedometer (steps, } \\
\text { physical activity) }\end{array}$ & $\begin{array}{l}\text { Zip } \\
\text { (Fitbit) }\end{array}$ & Right waist & $\begin{array}{l}\text { Steps/day, } \\
\text { MVPA min/ } \\
\text { day }\end{array}$ & $\begin{array}{l}\text { Spearman CC: } 0.91 \text { (ActiGraph } \\
\text { steps), } 0.86 \text { (ActiGraph MVPA), } \\
0.91 \text { (Yamax steps) }\end{array}$ \\
\hline
\end{tabular}

Wrist

Steps and

Pearson CC: treadmill walking

(Jawbone)

kilocalories (0.98 steps, 0.87 kilocalories)

keadmill running (0.98 steps, 0.69 kilocalories), elliptical (0.99 steps, 0.40 kilocalories), agility ( 0.34

min minute, mph miles per hour, MVPA moderate to vigorous physical activity, SD standard deviation, TST total sleep time, WASO wake after sleep onset 
participants, they found that the hip- and pocket-worn One generally over-estimated distance at the slower speeds $(0.90-1.33 \mathrm{~m} / \mathrm{s})$, but under-estimated at faster speeds $(1.78 \mathrm{~m} / \mathrm{s})[43]$.

\section{Validity for physical activity}

The criterion measures for two studies exploring physical activity relied on other accelerometers (ActiGraph GT3X [44] and ActiGraph GT3X+ [33], both using Freedson et al. cutpoints [49], and Body Media SenseWear [33]). Based on 42 participants wearing the Zip for 1 week during waking hours, moderate-to-vigorous physical activity showed almost perfect correlation with an accelerometer (Spearman CC 0.86) [44]. However, in another study of 21 participants wearing the Zip, One, and UP for 2 days without restrictions, compared to an accelerometer the trackers generally overcounted minutes of moderate-to-vigorous physical activity (mean absolute difference 89.8, 58.6, $18.0 \mathrm{~min} /$ day, respectively and intraclass $\mathrm{CC} 0.36,0.46,0.70$, respectively) [33].

\section{Validity for energy expenditure}

The criterion measures for energy expenditure assessed in kilocalories was indirect calorimetry $[15,29,34,35$, 39, 40, 45], direct calorimetry [31], accelerometry (ActiGraph GT3X+ with a conversion equation [50] to estimate kilocalories [35] and BodyMedia SenseWear [33]), and self-reported data using a questionnaire [41]. Generally, regardless of the criterion used, energy expenditure was under-estimated for the Classic [29, 31, 39, 41], One [33, 35], Flex, Ultra [29, 34] (for running, elliptical, and agility drills [40]), Zip [33, 35], UP [33, 35](for agility drills [40]), and UP24 [45]. When correlations were reported, they ranged widely [15, 29, 34, 35, 45]. A few studies indicated energy expenditure was over-estimated compared to indirect calorimetry: the Ultra during walking [40], the Zip across a variety of laboratory-based activities [35], the Flex during several combined activities (sedentary, aerobic, and resistance exercises) [45], and the UP during running [40].

\section{Validity for sleep}

Five studies explored the validity of sleep measures, four using polysomnography (PSG) [37, 38, 47, 48] and the other using the BodyMedia SenseWear device [33] as the criterion. Compared to PSG, the Classic [38], Ultra [37], and UP [47, 48] over-estimated total sleep time and sleep efficiency and under-estimated wake after sleep onset, resulting in high sensitivity and poor specificity. However, for the Ultra when using the sensitive mode setting, total sleep time and sleep efficiency were under-estimated and wake after sleep onset was over-estimated. In a study of 21 adults wearing the One and UP for 2 days without restrictions, compared to an accelerometer the trackers generally over-estimated time in sleep (mean absolute difference 23.0, $22.0 \mathrm{~min} /$ day, respectively and intraclass CC 0.90, 0.85, respectively) [33].

\section{Reliability}

No study reported on the intradevice or interdevice reliability of the Jawbone, or the intradevice reliability of the Fitbit. Seven studies reported on the interdevice reliability of several Fitbit trackers (Table 5), with sample sizes ranging from one [32, 36] to 30 [43]. Four studies were laboratory-based focusing solely on locomotion on the treadmill [15, 29, 36, 43], two studies were laboratorybased requiring monitoring with a PSG $[37,38]$, and one study was field-based [32]. For any Fitbit tracker, interdevice reliability was reported from five studies on steps $[15,29,32,36,43]$, one study on distance [43], no studies on physical activity, two studies on energy expenditure [15, 29], and two studies on sleep [37, 38]. The following sections detail the reliability results for each of the five measures.

\section{Reliability for steps}

Comparing two different hip-worn trackers for 16 to 23 participants during treadmill walking and running, the intraclass $\mathrm{CC}$ was substantial to almost perfect for steps taken for the Classic (range 0.86-0.91) and the Ultra (range 0.76-0.99) [29]. In another study, during six treadmill walking trials of 20 steps by one researcher, three hip-worn Ultras were compared and all trackers read within $5 \%$ of each other [36]. In a field-based study of 10 hip-worn Ultras all worn by the same person at the same time for 8 days, the median intraclass $\mathrm{CC}$ was 0.90 for steps/minute, 1.00 for steps/hour, and 1.00 for steps/day, and comparing across trackers, the maximum difference was only $3.3 \%$ [32].

Comparing three hip-worn Ones worn by 23 participants during treadmill walking and running, the Pearson $\mathrm{CC}$ between the left and right hip, as well as both right hips, was almost perfect for steps (0.99 and 0.99, respectively) [15]. In another study, 30 participants wore three Ones on their hips and front pants pocket while walking or running at five different speeds on the treadmill and correlation for steps was almost perfect when comparing across trackers (intraclass CC 0.95-1.00) [43]. Lastly, comparing two wrist-worn Flex trackers worn by 23 participants during treadmill walking and running, the Pearson CC between the left and right wrist was almost perfect for steps (0.90) [15].

\section{Reliability for distance}

In the only study of reliability assessment of distance, 30 participants wore three Ones on their hips and front 
Table 5 Fitbit and Jawbone reliability studies (listed by author's last name and publication year)

\begin{tabular}{|c|c|c|c|c|c|c|c|c|}
\hline \multirow[b]{2}{*}{ Author (year) } & \multicolumn{2}{|c|}{$\begin{array}{l}\text { Sample } \\
\text { characteristics }\end{array}$} & \multicolumn{2}{|l|}{ Tracker wearing protocol } & \multicolumn{3}{|c|}{ Measurements } & \multirow[t]{2}{*}{ Interdevice reliability results } \\
\hline & $n$ & $\%$ female & Activity & $\begin{array}{l}\text { Lab/ } \\
\text { field }\end{array}$ & Type & Placement & Measures & \\
\hline \multirow[t]{2}{*}{$\begin{array}{l}\text { Adam Noah et. } \\
\text { al (2013) [29] }\end{array}$} & 16 & 38 & \multirow{2}{*}{$\begin{array}{l}\text { Treadmill walking }(3.5 \mathrm{mph}) \text {, walking } \\
\text { with incline ( } 3.5 \mathrm{mph} \text { at } 5 \%) \text {, jogging } \\
(5.5 \mathrm{mph}) \text {, and stair stepping ( } 30.5 \\
\text { centimeter step at } 96 \text { beats/min) }\end{array}$} & \multirow[t]{2}{*}{ Lab } & $\begin{array}{l}\text { Ultra } \\
\text { (Fitbit) }\end{array}$ & Waist (1 on each side) & $\begin{array}{l}\text { Steps/min, } \\
\text { kilocalories/min }\end{array}$ & $\begin{array}{l}\text { ICC comparing } 2 \text { different devices worn at once: } \\
\text { range } 0.76-0.99 \text { (steps), range } 0.91-0.97 \text { (kilocalories) }\end{array}$ \\
\hline & 23 & 43 & & & $\begin{array}{l}\text { Classic } \\
\text { (Fitbit) }\end{array}$ & Waist (1 on each side) & $\begin{array}{l}\text { Steps/min, } \\
\text { kilocalories/min }\end{array}$ & $\begin{array}{l}\text { Comparing } 2 \text { different devices worn at once: } \\
\mathrm{ICC}=\text { average } 0.88 \text {, range } 0.86-0.91 \text { (steps); } \\
\text { average } 0.87 \text {, range } 0.74-0.92 \text { (kilocalories) }\end{array}$ \\
\hline \multirow[t]{2}{*}{ Diaz (2015) [15] } & \multirow[t]{2}{*}{23} & \multirow[t]{2}{*}{57} & \multirow[t]{2}{*}{$\begin{array}{l}6 \text { min each of treadmill walking } \\
(1.9 \mathrm{mph}, 3.0 \mathrm{mph}, 4.0 \mathrm{mph} \text { ) and } \\
\text { jogging (5.2 mph) }\end{array}$} & \multirow[t]{2}{*}{ Lab } & $\begin{array}{l}\text { One } \\
\text { (Fitbit) }\end{array}$ & 2 on right hip, 1 on left hip & $\begin{array}{l}\text { Steps/min, } \\
\text { kilocalories/min }\end{array}$ & $\begin{array}{l}\text { Pearson CC left and right hips: } 0.99 \text { (steps), } \\
0.97 \text { (kilocalories); Pearson CC two right hip devices: } \\
0.99 \text { (steps), } 0.96 \text { (kilocalories) }\end{array}$ \\
\hline & & & & & $\begin{array}{l}\text { Flex } \\
\text { (Fitbit) }\end{array}$ & 1 on each wrist & $\begin{array}{l}\text { Steps/min, } \\
\text { kilocalories/min }\end{array}$ & $\begin{array}{l}\text { Pearson CC left and right wrists: } 0.90 \text { (steps), } \\
0.95 \text { (kilocalories) }\end{array}$ \\
\hline $\begin{array}{l}\text { Dontje (2015) } \\
\text { [32] }\end{array}$ & 1 & 0 & $\begin{array}{l}8 \text { consecutive days excluding sleep } \\
\text { and water-based activities }\end{array}$ & Field & $\begin{array}{l}\text { Ultra } \\
\text { (Fitbit) }\end{array}$ & $\begin{array}{l}5 \text { over left pants pocket, } 5 \text { over } \\
\text { right pants pocket }\end{array}$ & $\begin{array}{l}\text { Steps/min, } \\
\text { steps/hour, } \\
\text { steps/day }\end{array}$ & $\begin{array}{l}10 \text { devices collected movement (yes vs no) across } \\
\text { minutes ( } 98 \% \text { ); two-way median ICC of absolute } \\
\text { agreement } 0.90 \text { (steps/min), } 1.00 \text { (steps/hour), } 1.00 \\
\text { (steps/day); concordance CC } 0.90 \text { (steps/min), } 1.00 \\
\text { (steps/hour), } 0.99 \text { (steps/day); from Bland-Altman } \\
\text { plots } 95 \% \text { of the measures were within the } \\
\text { boundaries of } 28 \text { steps above and below the mean } \\
\text { difference; maximum difference for all devices was } 3.3 \%\end{array}$ \\
\hline $\begin{array}{l}\text { Mammen (2012) } \\
{[36]}\end{array}$ & 1 & 0 & $\begin{array}{l}6 \text { trials were performed while the } \\
\text { researcher wore the devices and } \\
\text { walked } 20 \text { steps }\end{array}$ & Lab & $\begin{array}{l}\text { Ultra } \\
\text { (Fitbit) }\end{array}$ & $\begin{array}{l}3 \text { trials on right hip, } 3 \text { trials on } \\
\text { left hip }\end{array}$ & Steps/trial & All trackers were within $+/-5 \%$ of each other \\
\hline $\begin{array}{l}\text { Meltzer (2015) } \\
\text { [37] }\end{array}$ & 9 & $\begin{array}{l}\text { Not } \\
\text { reported }\end{array}$ & 1 night's sleep & Lab & $\begin{array}{l}\text { Ultra } \\
\text { (Fitbit) }\end{array}$ & 2 on nondominant wrist & $\begin{array}{l}\text { TST, sleep } \\
\text { efficiency }\end{array}$ & $\begin{array}{l}\text { Among } n=7: \text { no differences between trackers for TST } \\
(468.7 \text { vs. } 471.1 \text { min normal mode; } 300.4 \text { vs. } 289.9 \text { min } \\
\text { sensitive mode) or sleep efficiency ( } 92.9 \% \text { vs. } 93.3 \% \\
\text { normal mode; } 59.4 \% \text { vs. } 57.4 \% \text { sensitive mode) }\end{array}$ \\
\hline $\begin{array}{l}\text { Montgomery- } \\
\text { Downs (2012) } \\
\text { [38] }\end{array}$ & 3 & $\begin{array}{l}\text { Not } \\
\text { reported }\end{array}$ & 1 night's sleep & Lab & $\begin{array}{l}\text { Classic } \\
\text { (Fitbit) }\end{array}$ & 2 on nondominant wrist & Sleep vs. wake & $\begin{array}{l}3 \text { participant's recorded } 96.5 \%, 99.1 \% \text {, and } 97.6 \% \\
\text { agreement at 1-minute epochs }\end{array}$ \\
\hline $\begin{array}{l}\text { Takacs (2014) } \\
{[43]}\end{array}$ & 30 & 50 & $\begin{array}{l}5 \text { min each of treadmill walking }(0.90 \\
1.12,1.33,1.54,1.78 \text { meters/second })\end{array}$ & Lab & $\begin{array}{l}\text { One } \\
\text { (Fitbit) }\end{array}$ & $\begin{array}{l}1 \text { on the waist at each hip, } 1 \text { in } \\
\text { front pocket of the dominant } \\
\text { leg }\end{array}$ & $\begin{array}{l}\text { Steps/trial, } \\
\text { distance/trial }\end{array}$ & $\begin{array}{l}\text { Across } 5 \text { treadmill speeds ICC: range } 0.95-1.00 \text { (steps), } \\
\text { range } 0.90-0.99 \text { (distance) }\end{array}$ \\
\hline
\end{tabular}


pants pocket while walking or running at five different speeds on the treadmill and the correlation was almost perfect for distance measurements across trackers (intraclass CC 0.90-0.99) [43].

\section{Reliability for energy expenditure}

Comparing two different hip-worn trackers for 16-23 participants during treadmill walking and running, the intraclass CC was substantial to almost perfect for kilocalories expended for the Classic (range 0.74-0.92) and the Ultra (range 0.91-0.97) [29]. Comparing three hipworn Ones worn by 23 participants during treadmill walking and running, the Pearson CC between the left and right hip, as well as both right hips, was almost perfect for kilocalories expended (0.97 and 0.96, respectively) [15]. These same participants wore two Flex trackers on their wrists during treadmill walking and running that had almost perfect correlation for kilocalories expended (0.95) [15].

\section{Reliability for sleep}

Three participants wore two Classics overnight and recorded almost perfect levels of agreement (96.5-99.1\%) to classify whether the minute-level data was a sleep or wake minute [38]. Similarly, nine youth participants wore two Ultras on their wrist overnight, with data available for seven participants (one pair did not record and one pair had significant discrepancies between readings) [37]. They found similar readings for total sleep time and sleep efficiency for either the normal or sensitive mode.

\section{Feasibility}

Feasibility assessment was abstracted for the 22 studies in this review. In total, seven of 18 studies reported on missing or lost data, with the lab-based studies less likely to report it than the field-based studies. For the lab measurements, Case et al. [30] indicated $1.4 \%$ of data were missing from all tested trackers due to not properly setting them to record steps, Dannecker et al. [31] indicated incomplete data on two of 19 participants, and Gusmer et al. [34] excluded six of 32 participants because ActiGraph step counts were about half of the Ultra step counts (they note this is most likely an ActiGraph failure). For one night of recording in the sleep laboratory, Meltzer et al. [37] reported missing data for 14 of 63 participants to assess validity, due to data not recording for the Ultra $(n=12)$ and corrupted PSG files $(n=2)$.

For a field-based study of 21 participants during 2 days of wear some data were lost: moderate-to-vigorous physical activity ( $n=7$ due to data extraction of the One and the Zip (i.e., certain data were only available for a limited amount of time), $n=1$ Zip malfunction), steps ( $n=1$ Zip malfunction), energy expenditure ( $n=1$ Zip malfunction), and sleep ( $n=2$ participant error for the One) [33]. In a second field-based study enrolling adults > $=60$ years of age, authors excluded five of 15 participants because they had difficulty with the Classic over the 10day period (two lost the tracker and three failed to plug it into the wireless base to transmit data) [41]. In a separate field-based study, the Zip was worn over 1 week and five of 47 participants had at least some missing data [44].

\section{Discussion}

This review summarized the evidence for validity and reliability of activity trackers, identifying 22 studies published since 2012. While conducting this review, we learned how the trackers can be set-up to improve upon off-the-shelf accuracy. Those testing and wearing the trackers are encouraged to consider several tips to potentially improve the trackers' performance (Table 6).

\section{Validity and reliability}

From this review, we found the validity (Fitbit and Jawbone) and interdevice reliability (Fitbit) of steps counts was generally high, particularly during laboratory-based treadmill tests. When errors were higher, the direction tended to be an under-estimation of steps by the tracker compared to the criterion. This may be particularly problematic at slow walking speeds, similar to findings when testing pedometers [51]. Specifically for steps, if the option is available to set stride length, this should improve accuracy (Table 6). Hip-worn trackers generally performed better at counting steps than trackers worn elsewhere on the body, although Mammen et al. [36] suggests moving the placement from the hip if being worn by an older adult with slower gait speed. Only one study assessed the validity and reliability of distance walked, finding that while reliability was high, distance was over-estimated at slower speeds and underestimated at faster speeds [43].

Compared to other accelerometers, one study indicated that the trackers generally over-counted moderateto-vigorous physical activity, with some large differences found (mean 0.3, 1.0, and $1.5 \mathrm{~h} /$ day for the UP, One, and Zip, respectively) [33]. However, another study indicated higher agreement [44]. It may be that the cutpoints [49] used to define moderate-to-vigorous physical activity in both studies were set too high, particularly for older or inactive adults. The reliability of physical activity measurement has not been tested in any study.

From 10 adult studies, we found that although interdevice reliability of energy expenditure was high, the validity of the tracker was lower. When reported, the CC generally ranged from moderate to substantial agreement. Across trackers, many studies indicated that the 
Table 6 Strategies to improve the activity tracker accuracy for steps, distance, physical activity, energy expenditure, and sleep

\begin{tabular}{ll} 
Instruction & Explanation \\
\hline Wear the tracker in the same position & While wearing the activity tracker in the same position daily may be obvious for the wrist-based
\end{tabular}

each day

Enter your details and sync

trackers, those worn on a pocket, bra, or hip could vary in accuracy depending on location. Trackers are more accurate when worn close to the body ${ }^{a}$. For free-living research studies, the wearing location should be standardized and communicated to participants.

At initial set-up, users should accurately enter height, weight, gender, and age into the application and sync it to the tracker. For example, these characteristics, as well as heart rate if available, are used by the Fitbit to calculate energy expenditure ${ }^{b}$. Related to this, if body weight meaningfully changes, then updating the tracker with the new weight would be important.

For wrist-worn trackers, indicate if wearing In the software set-up, indicate if possible whether the wrist-worn tracker is being worn on the domit on the domin Inant or non-dominant hand. For Jawbone, trackers worn on the non-dominant wrist may be more accurate ${ }^{c}$, probably because the non-dominant hand is less active than the dominant hand, so it provides a better representation of overall body movement. Fitbit indicates that using the nondominant hand setting increases sensitivity of step counting and can be used if the tracker is under counting steps ${ }^{d}$.

Calibrate stride length

Calibrating stride length may improve distance measures. In our review, only one study indicated that this was performed [34]. Fitbit indicates a default stride length is used otherwise, based on height and gender ${ }^{\mathrm{e}}$. Jawbone also provides information for calibrationf.

Use add-on features and obtain updates

Using add-on features and obtaining updates might become more important since future iterations of algorithms to calculate physical activity or energy expenditure may use new features, such as heart rate and respiration. For example, Fitbit indicates that trackers with heart rate better recogniz "active minutes" for physical activities that do not incorporate stepping, such as weight lifting or rowinge.

Add more information via the diary or journal function

Providing information to the tracker on the specific physical activity being performed can help the tracker learn what activities look like for the individual. This is particularly important if the algorithms used by the activity tracker rely on machine learning techniques.

Interact with the sleep mode settings

Interacting with the sleep mode settings may help the tracker learn if the user is sleeping, napping, or awake. Fitbit indicates that the normal mode counts significant movements as being awake and is appropriate for most users, while the sensitive setting will record nearly all movements as time awake ${ }^{f}$.
Web Links: accessed 10/14/2015

ahttp://help.fitbit.com/articles/en_US/Help_article/

How-do-I-wear-my-Zip/

bhttp//helpfitbit.com/articles/en_US/Help article/ How-does-Fitbit-know-how-many-calories-I-veburned

${ }^{c}$ https://jawbone.com/up/faq

dhttp://help.fitbit.com/articles/en_US/Help_article/ How-accurate-is-my-Surge

${ }^{d}$ http://help.fitbit.com/articles/en_US/Help_article/ How-do-I-measure-and-adjust-my-stride-length

ehttps:/help.jawbone.com/articles/en US/ PKB_Article/424

fhttps://help.fitbit.com/articles/en_US/Help_article/ What-are-very-active-minutes/

ghttp://help.fitbit.com/articles/en_US/Help_article/ Sleep-tracking-FAQs\#Whatisthedifference 
bias in mis-reporting was often an under-estimation of energy expended.

For sleep among youth and adults, despite high reliability, the trackers evaluated generally over-estimated total sleep time [33, 37, 38, 47, 48], and when tested against PSG the trackers over-estimated sleep efficiency and under-estimated wake after sleep onset [37, 38, 47, 48]. These findings are similar to other studies of accelerometry, in which the devices are highly sensitive but do not accurately detect periods of wake before and during sleep [52]. However, for one tracker the sensitive mode setting was tested, which under-estimated total sleep time and sleep efficiency and over-estimated wake after sleep onset [37]. Work is needed to improve the validity of sleep measurement with these trackers, particularly when using them for only one or two nights of testing [38]. It may be that newer trackers will perform better if they "learn" when the person is asleep, awake, or napping (Table 6).

\section{Feasibility}

Seven of 22 studies reported on missing or lost data, ranging from approximately 1.4 to $22.2 \%$ for laboratorybased studies and 10.6 to $33.3 \%$ for field-based studies. Some of the lost data was attributable to the validation criterion measure and not the trackers, and other lost data were attributable to researcher error and not participant error. Even so, researchers should anticipate data loss based on these findings. Future studies should report missing data and the reason for the loss. One study in this review [44] and others not included [4, 8, $19,53]$ report relatively high acceptability in wearing the trackers. This type of information may help with understanding reasons for missing data in field-based studies, particularly if they occur over long time periods.

\section{For the companies}

Through this review, we identified three recommendations manufacturers can contribute to enhance the use of the trackers for research. First, the trackers contain firmware, defined as an electronic component with embedded software to control the tracker. Firmware can be updated by the company at any time; when the tracker is synched, the new software is updated. These software changes can influence the measurement properties in either positive or negative ways, and can change what might have been previously confirmed or published. Firmware may fix bugs or add features to the tracker, or it may change how variables are calculated. However, many other changes take place, which the consumer cannot detect [54]. As an alternative, the company supporting ActiGraph accelerometers currently makes firmware updates available to the public via their website, allowing researchers to assess those changes for impact on the measurement properties of the accelerometer [55, 56]. A similar standard operating procedure would be a beneficial approach for researchers using these trackers.

Second, Jawbone UP3 and UP4 trackers include bioelectric impedance, with corresponding measures of heart rate and respiration, and both skin and ambient temperatures. Additionally, some of the newer Fitbit trackers include GPS (Surge) and optical heart rate sensors (Surge and Charge HR). With these enhancements, the companies seemingly have the tools to determine whether the tracker is being worn (e.g., adherence) and whether it is being worn by the same individual (e.g., one body authentication) [8]. It would be beneficial if the companies derived an indicator of wear and made this available on a minute-by-minute level, corresponding to other available data. Currently, neither the Jawbone nor Fitbit indicate the time worn, which could impact all metrics studied in this review.

Third, the companies could allow access to more data that are collected. At present, the trackers provide users with only a subset of data that is actually collected. The companies control the output available, making the daylevel summary variables the easiest to obtain. For example, despite capturing GPS and heart rate on two trackers, Fitbit currently limits the export of these full datasets. Furthermore, the resulting output is derived through proprietary algorithms that may change over time and with new features. In all likelihood, based on the performance of the trackers found in this review, these algorithms are supported through machine learning techniques. At a minimum, it would be helpful for companies to reveal what pieces of data are being used by the trackers to calculate each output measure. For example, Jawbone indicates that height, weight, gender, age, and heart rate, if available, are used to calculate physical activity [14].

\section{Future research}

In total, Fitbit offered at least 9 trackers since 2008 and Jawbone offered at least 6 trackers since 2011. Until we understand if the specifications within a company's family of trackers are similar, researchers should confirm the validity and reliability of new trackers. Moreover, an argument could be made to test any new tracker, even if the company confirms similar hardware and software processes. With time, the trackers offer more features through enhancements made to the trackers (Table 1). Each new tracker feature needs testing for reliability, validity, and usability. Specific types of activities should also be tested, similar to the study by Sasaki et al. [39]. While this review focused on steps, distance, physical activity, energy expenditure, and sleep, other features to test include number of stair flights taken, heart rate, respiration, location via GPS technology, skin temperature, and ambient temperature. 
Exploring the measurement properties of the trackers in a wide variety of populations would also be important in both laboratory and field settings. Free-living activities may better reflect the true accuracy of the tracker, because daily activities include a considerable amount of upper body movement that may or may not be accurately captured by the trackers [35]. Currently, the review only identified two studies that included children [37, 48]. Researchers mostly tested the trackers in middleaged adult populations with normal BMI. Since studies of pedometers indicate lower accuracy among participants with higher BMI [57], it would be prudent to test various trackers types and locations among participants with higher BMI [43].

Moreover, with the proliferation of trackers, researchers would benefit from an evidence-based position statement on the properties necessary to consider a tracker valid and reliable [38]. Guidance on equivalency of accelerometers exists [58], but this review found a variety of statistical methods applied to the data and interpreted slightly differently across studies. Those who conduct future studies on the measurement properties of the trackers should be sure to initialize the tracker properly and indicate in the publication how this was done so others can replicate the process. Providing the specific tracker type, date purchased, and date tested would also be important.

Notably there were no reliability studies of any Jawbone tracker or the Fitbit Zip, and no intradevice reliability studies of any trackers. While more field-based studies are needed, the laboratory studies indicated high interdevice reliability for measuring steps, energy expenditure, and sleep. Only one study assessed distance, also finding high interdevice reliability during treadmill walking and running [43]. It would be ideal practice for all studies or programs to test the trackers for reliability before deploying them for either measurement or intervention.

While not reviewed here, researchers should also consider issues related to privacy and informed consent with activity trackers and smart phone applications [59, 60]. Since the trackers can measure and store data for long periods of time passively, providing informed consent takes on new meaning with the extended time period, locational information, and re-use of data in successive analyses. Users should also be aware that the companies access and use the data that are entered and collected [61]. Recent examples include an indication of the states with the most steps by Fitbit users [62] and the impact of the prior day's sleep and steps taken on self-reported mood by Jawbone users [63].

\section{Limitations}

Our review has several limitations. The literature on activity trackers is rapidly building and it is possible that studies were missed despite our best efforts. We encountered some challenges with comparing across studies, due to varying methods and reported results. The findings should be viewed in light of the variety of study protocols and methodology.

When we began the systematic review in fall 2014, we were guided by the most recent market data available at that time, indicating that Fitbit and Jawbone represented the majority of the consumer market [2]. In June 2015, market share from the first quarter sales in 2015 indicated the top five vendors were Fitbit (34\%), Xiaomi (25\%), Garmin (6\%), Samsung (5\%), and Jawbone (4\%) [64]. There is a built-in time lag between manufacturing and sale of activity trackers to use in the research laboratory and field. Thus, some activity trackers that are currently available to consumers were not represented in this review, but should be considered as future studies accumulate on new devices and brands.

\section{Conclusions}

This systematic review of 22 studies included assessments of five Fitbit and two Jawbone trackers, focusing on validity and reliability of steps, distance, physical activity, energy expenditure, and sleep. No single specific tracker had a complete assessment across the five measures. This review also described several ways to improve the trackers' accuracy, offered recommendations to companies selling the trackers, and identified future areas of research. Generally, the review indicated higher validity of steps, fewer studies on distance and physical activity, and lower validity for energy expenditure and sleep. These studies also indicated high interdevice reliability for steps, energy expenditure, and sleep for certain Fitbit models, but with no studies on the Jawbone. As new activity trackers and features are introduced to the market, documentation of the measurement properties can guide their use in research settings.

\section{Additional file}

Additional file 1: Flow of article selection using the PRISMA schematic (Liberati et al., 2009 [27]; Moher et al., 2009 [28]). (PDF $62 \mathrm{~kb}$ )

\section{Abbreviations}

BMI: Body mass index; CC: Correlation coefficient; GPS: Global positioning system; PRISMA: Preferred Reporting Items for Systematic Reviews and MetaAnalyses; PSG: Polysomnography; SD: Standard deviation; US: United States.

Competing interests

The authors declare that they have no competing interests.

Authors' contributions

KRE developed the aims of the study, helped conduct the literature review, coded all articles, contacted authors for missing information, and drafted the paper. All remaining authors provided critical feedback on several earlier drafts of the paper. MMG also conducted the final literature review and coded all articles. All authors read and approved the final manuscript. 


\section{Acknowledgment}

We thank Sonia Grego, Sara Satinsky, and the anonymous reviewers for comments on earlier drafts of this paper. We also thank the authors of the reviewed studies for responding to our requests for further information and clarification. This work was supported, in part, by RTI International through the RTI University Scholars Program and iSHARE. The content is solely the responsibility of the authors and does not necessarily represent the official views of RTI International.

\section{Received: 5 August 2015 Accepted: 4 December 2015}

\section{Published online: 18 December 2015}

\section{References}

1. Almalki M, Gray K, Sanchez FM. The use of self-quantification systems for personal health information: big data management activities and prospects. Health Information Science Systems. 2015;3(Suppl 1 HISA Big Data in Biomedicine and Healthcare 2013 Con):S1. doi: 10.1186/2047-2501-3-S1-S1.

2. Danova T. Just 3.3 million fitness trackers were sold in the US in the past year. Business Insider 2014. http://www.businessinsider.com/33-million-fitnesstrackers-were-sold-in-the-us-in-the-past-year-2014-5. Accessed March 2, 2015.

3. Lyons EJ, Lewis ZH, Mayrsohn BG, Rowland JL. Behavior change techniques implemented in electronic lifestyle activity monitors: A systematic content analysis. J Med Internet Res. 2014;16(8):e192. doi:10.2196/jmir.3469.

4. Cadmus-Bertram LA, Marcus BH, Patterson RE, Parker BA, Morey BL. Randomized trial of a Fitbit-Based physical activity intervention for women Am J Prev Med. 2015:49(3):414-8.

5. Michie S, Ashford S, Sniehotta FF, Dombrowski SU, Bishop A, French DP. A refined taxonomy of behaviour change techniques to help people change their physical activity and healthy eating behaviours: The CALO-RE taxonomy. Psych Health. 2011;26(11):1479-98.

6. Fox S, Duggan M. Tracking for Health. Pew Research Center, Pew Internet and American Life Project. 2013. http://pewinternet.org/Reports/2013/ Tracking-for-Health.aspx. Accessed October 9, 2015.

7. Bentley F, Tollmar K, Stephenson P, Levy L, Jones B, Robertson S, et al. Health mashups: Presenting statistical patterns between well-being data and context in natural language to promote behavior change. ACM Trans Comput-Hum Interact. 2013:20(5):1-25.

8. Kurti AN, Dallery J. Internet-based contingency management increases walking in sedentary adults. J Appl Behav Anal. 2013:46(3):568-81.

9. Washington WD, Banna KM, Gibson AL. Preliminary efficacy of prize-based contingency management to increase activity levels in healthy adults. J Appl Behav Anal. 2014;47(2):231-45.

10. Thompson WG, Kuhle CL, Koepp GA, McCrady-Spitzer SK, Levine JA. "Go4Life" exercise counseling, accelerometer feedback, and activity levels in older people. Arch Gerontol Geriatr. 2014;58(3):314-9.

11. Wang JB, Cadmus-Bertram LA, Natarajan L, White MM, Madanat H, Nichols $J$ F, et al. Wearable sensor/device (Fitbit One) and SMS text-messaging prompts to increase physical activity in overweight and obese adults: A randomized controlled trial. Telemed J E-Health. 2015;21(10):782-92.

12. Hayes LB, Van Camp CM. Increasing physical activity of children during school recess. J Appl Behav Anal. 2015;48(3):690-5

13. Fitbit Inc. How accurate are Fitbit trackers? 2015. http://help.fitbit.com/ articles/en_US/Help_article/How-accurate-are-Fitbit-trackers. Accessed June 16, 2015.

14. Jawbone. Jawbone UP: Activity Data Issues. 2015. https://help.jawbone.com/ articles/en_US/PKB_Article/activity-data-issues-up. Accessed June 16, 2015.

15. Diaz KM, Krupka DJ, Chang MJ, Peacock J, Ma Y, Goldsmith J, et al. Fitbit: An accurate and reliable device for wireless physical activity tracking. Intl J Cardiol. 2015;185:138-40.

16. Klassen TD, Eng JJ, Chan C, Hassall Z, Lim S, Louie R, et al. Step count monitor for individuals post-stroke: Accuracy of the Fitbit One. Stroke. 2014; 45(12):e261.

17. Perez-Macias JM, Jimison H, Korhonen I, Pavel M. Comparative assessment of sleep quality estimates using home monitoring technology. Conference proceedings: Annual International Conference of the IEEE Engineering in Medicine and Biology Society IEEE Engineering in Medicine and Biology Society Annual Conference. 2014; 2014:4979-82. doi: 10.1109/embc.2014 6944742.

18. Fulk GD, Combs SA, Danks KA, Nirider CD, Raja B, Reisman DS. Accuracy of 2 activity monitors in detecting steps in people with stroke and traumatic brain injury. Phys Ther. 2014;94(2):222-9.
19. Vooijs M, Alpay LL, Snoeck-Stroband JB, Beerthuizen T, Siemonsma PC, Abbink JJ, et al. Validity and usability of low-cost accelerometers for internet-based self-monitoring of physical activity in patients with chronic obstructive pulmonary disease. Interactive J Med Res. 2014;3(4):e14. doi:10. 2196/ijmr.3056.

20. Albert MV, Deeny S, McCarthy C, Valentin J, Jayaraman A. Monitoring daily function in persons with transfemoral amputations using a commercial activity monitor: A feasibility study. PM \& R: J Inj Funct Rehabil. 2014;6(12): 1120-7. doi:10.1016/j.pmrj.2014.06.006.

21. Naslund JA, Aschbrenner KA, Barre LK, Bartels SJ. Feasibility of popular mhealth technologies for activity tracking among individuals with serious mental illness. Telemed J E-Health. 2015;21(3):213-6.

22. Phillips $\amalg$, Petroski GF, Markis NE. A comparison of accelerometer accuracy in older adults. Res Gerontol Nursing. 2015: 1-7. doi:10.3928/1940492120150429-03.

23. Lauritzen J, Munoz A, Luis Sevillano J, Civit A. The usefulness of activity trackers in elderly with reduced mobility: A case study. Stud Health Technol Inform. 2013;192:759-62

24. De Vries SI, Van Hirtum HW, Bakker I, Hopman-Rock M, Hirasing RA, Van Mechelen W. Validity and reproducibility of motion sensors in youth: A systematic update. Med Sci Sports Exerc. 2009;41(4):818-27.

25. Higgins PA, Straub AJ. Understanding the error of our ways: Mapping the concepts of validity and reliability. Nurs Outlook. 2006;54(1):23-9.

26. Landis J, Koch $\mathrm{G}$. The measurement of observer agreement for categorical data. Biometrics. 1977:33:159-74.

27. Liberati A, Altman DG, Tetzlaff J, Mulrow C, Gotzsche PC, loannidis JP, et al. The PRISMA statement for reporting systematic reviews and meta-analyses of studies that evaluate health care interventions: Explanation and elaboration. PLoS Med. 2009;6(7):e1000100. doi:10.1371/journal.pmed. 1000100.

28. Moher D, Liberati A, Tetzlaff J, Altman DG, Group P. Preferred reporting items for systematic reviews and meta-analyses: The PRISMA statement. PLoS Med. 2009;6(7):e1000097. doi:10.1371/journal.pmed.1000097.

29. Adam Noah J, Spierer DK, Gu J, Bronner S. Comparison of steps and energy expenditure assessment in adults of Fitbit Tracker and Ultra to the Actical and indirect calorimetry. J Med Eng Tech. 2013;37(7):456-62.

30. Case MA, Burwick HA, Volpp KG, Patel MS. Accuracy of smartphone applications and wearable devices for tracking physical activity data. JAMA. 2015;313(6):625-6.

31. Dannecker KL, Sazonova NA, Melanson EL, Sazonov ES, Browning RC. A comparison of energy expenditure estimation of several physical activity monitors. Med Sci Sports Exerc. 2013;45(11):2105-12.

32. Dontje ML, de Groot M, Lengton RR, van der Schans CP, Krijnen WP. Measuring steps with the Fitbit activity tracker: An inter-device reliability study. J Med Eng Tech. 2015:39(5):286-90.

33. Ferguson T, Rowlands AV, Olds T, Maher C. The validity of consumer-level, activity monitors in healthy adults worn in free-living conditions: A crosssectional study. Intl J Behav Nutr Phys Act. 2015;12:42. doi:10.1186/s12966015-0201-9.

34. Gusmer R, Bosch T, Watkins A, Ostrem J, Dengel D. Comparison of Fitbit Ultra to ActiGraph GT1M for assessment of physical activity in young adults during treadmill walking. Open Sports Med J. 2014;8:11-5.

35. Lee JM, Kim Y, Welk GJ. Validity of consumer-based physical activity monitors. Med Sci Sports Exerc. 2014;46(9):1840-8.

36. Mammen G, Gardiner S, Senthinathan A, McClemont L, Stone M, Faulkner G. Is this bit fit? Measuring the quality of the FitBit step-counter. Health Fit J Can. 2012:5(4):30-9.

37. Meltzer LJ, Hiruma LS, Avis K, Montgomery-Downs H, Valentin J. Comparison of a commercial accelerometer with polysomnography and actigraphy in children and adolescents. Sleep. 2015;38(8):1323-30.

38. Montgomery-Downs HE, Insana SP, Bond JA. Movement toward a novel activity monitoring device. Sleep Breath. 2012;16(3):913-7.

39. Sasaki JE, Hickey A, Mavilia M, Tedesco J, John D, Kozey Keadle S, et al. Validation of the Fitbit wireless activity tracker for prediction of energy expenditure. J Phys Act Health. 2015;12:149-54.

40. Stackpool CM, Porcari JP, Mikat RP, Gillette C, Foster C. The accuracy of various activity trackers in estimating steps taken and energy expenditure. J Fit Res. 2014;3(3):32-48.

41. Stahl ST, Insana SP. Caloric expenditure assessment among older adults: Criterion validity of a novel accelerometry device. J Health Psych. 2014; 19(11):1382-7. 
42. Storm FA, Heller BW, Mazza C. Step detection and activity recognition accuracy of seven physical activity monitors. PLoS ONE. 2015;10(3): e0118723. doi:10.1371/journal.pone.0118723.

43. Takacs J, Pollock CL, Guenther JR, Bahar M, Napier C, Hunt MA. Validation of the Fitbit One activity monitor device during treadmill walking. J Sci Med Sport. 2014;17(5):496-500.

44. Tully MA, McBride C, Heron L, Hunter RF. The validation of Fibit Zip physical activity monitor as a measure of free-living physical activity. BMC Res Notes. 2014;7:952. doi:10.1186/1756-0500-7-952.

45. Bai Y, Welk GJ, Nam YH, Lee JA, Lee JM, Kim Y et al. Comparison of consumer and research monitors under semistructured settings. Med Sci Sports Exercise. 2015, in press. doi:10.1249/MSS.0000000000000727.

46. Simpson LA, Eng JJ, Klassen TD, Lim SB, Louie DR, Parappilly B, et al. Capturing step counts at slow walking speeds in older adults: Comparison of ankle and waist placement of measuring device. J Rehabil Med. 2015; 47(9):830-5.

47. de Zambotti M, Claudatos S, Inkelis S, Colrain IM, Baker FC. Evaluation of a consumer fitness-tracking device to assess sleep in adults. Chronobiol Intl. 2015;32(7):1024-8.

48. de Zambotti M, Baker FC, Colrain IM. Validation of sleep-tracking technology compared with polysomnography in adolescents. Sleep. 2015;38(9):1461-8.

49. Freedson PS, Melanson E, Sirard J. Calibration of the computer science and applications, Inc. accelerometer. Med Sci Sports Exerc. 1998;30(5):777-81

50. Sasaki JE, John D, Freedson PS. Validation and comparison of ActiGraph activity monitors. J Sci Med Sport. 2011;14(5):411-6.

51. Crouter SE, Schneider PL, Karabulut M, Bassett Jr DR. Validity of 10 electronic pedometers for measuring steps, distance, and energy cost. Med Sci Sports Exerc. 2003;35(8):1455-60.

52. Meltzer LJ, Montgomery-Downs HE, Insana SP, Walsh CM. Use of actigraphy for assessment in pediatric sleep research. Sleep Med Rev. 2012;16(5):463-75.

53. Shih P, Han K, Poole E, Rosson M, Carroll J. Use and adoption challenges of wearable activity trackers. 2015. iConference 2015 Proceedings. https:// www.ideals.illinois.edu/handle/2142/73649. Accessed June 16, 2015.

54. Fitbit I. A brief look into how the Fitbit algorithms work. 2009.

55. John D, Freedson P. ActiGraph and Actical physical activity monitors: A peek under the hood. Med Sci Sport Exerc. 2012;44(1 Suppl 1):S86-9.

56. John D, Sasaki J, Hickey A, Mavilia M, Freedson PS. ActiGraph activity monitors: "The firmware effect". Med Sci Sport Exerc. 2014;46(4):834-9.

57. Crouter SE, Schneider PL, Bassett Jr DR. Spring-levered versus piezo-electric pedometer accuracy in overweight and obese adults. Med Sci Sport Exerc. 2005;37(10):1673-9.

58. Welk GJ, McClain J, Ainsworth BE. Protocols for evaluating equivalency of accelerometry-based activity monitors. Med Sci Sport Exerc. 2012;44(1 Suppl 1):S39-49.

59. King AC, Glanz K, Patrick K. Technologies to measure and modify physical activity and eating environments. Am J Prev Med. 2015;48(5):630-8.

60. de Montjoye YA, Hidalgo CA, Verleysen M, Blondel VD. Unique in the Crowd: The privacy bounds of human mobility. Sci Rep. 2013;3:1376. doi:10. 1038/srep01376.

61. Health Data Exploration Project. Personal Data for the Public Good: New Opportunities to Enrish Understanding of Individual and Population Health. 2014. http://www.rwjf.org/content/dam/farm/reports/reports/2014/ rwjf41 1080. Accessed October 9, 2015. Calit2, UC Irvine and UC San Diego.

62. Fitbit Inc. Weathering the weather. 2015. https://www.fitbit.com/ weathermap. Accessed October 9, 2015.

63. Mohan S. The Jawbone Blog: What makes people happy? We have the data. 2015. https://jawbone.com/blog/what-makes-people-happy/. Accessed October 9, 2015.

64. IDC. Wearable Market Remained Strong in the First Quarter Despite the Pending Debut of the Apple Watch, Says IDC. Press release from IDC on June 3, 2015. Based on the "IDC Worldwide Quarterly Wearable Tracker, June 2, 2015". 2015. http://www.idc.com/getdoc. jsp?containerld=prUS25658315. Accessed October 9, 2015.

\section{Submit your next manuscript to BioMed Central and we will help you at every step:}

- We accept pre-submission inquiries

- Our selector tool helps you to find the most relevant journal

- We provide round the clock customer support

- Convenient online submission

- Thorough peer review

- Inclusion in PubMed and all major indexing services

- Maximum visibility for your research

Submit your manuscript at www.biomedcentral.com/submit
Biomed Central 